\title{
Regulation of brain aging by neutral sphingomyelinase 2
}

Abbreviated title : nSMase2 and brain aging

Zhihui Zhu ${ }^{1}$, Zainuddin Quadri ${ }^{1,2}$, Simone M. Crivelli ${ }^{1}$, Ahmed Elsherbini ${ }^{1}$, Liping Zhang ${ }^{1,2}$,

Priyanka Tripathi ${ }^{1,2}$, Haiyan Qin ${ }^{1}$, Emily Roush ${ }^{1}$, Stefka D. Spassieva ${ }^{1}$, Mariana Nikolova-

Karakashian $^{1}$, Timothy S. McClintock ${ }^{1}$, and Erhard Bieberich ${ }^{1,2}$

${ }^{1}$ Department of Physiology, College of Medicine, University of Kentucky, Lexington, KY

${ }^{2}$ Veterans Affairs Medical Center, Lexington, KY 40502, United States

\section{Correspondence to:}

Dr. Erhard Bieberich

Department of Physiology, School of Medicine, University of Kentucky 800 Rose St. Room MS519. Lexington, KY 40536, Phone: 859-323-8079. Email: Erhard.bieberich@uky.edu

Number of pages: 35

Number of figures: 8 , tables: 3

Number of words for abstract (249), Introduction: (586), Discussion (1498 words):

Conflict of interest statement: The authors declare no competing financial interests.

\section{Acknowledgements}

This work was supported by NIH grants R01NS095215, R01AG034389, and R01AG064234, and the VA grant I01BX003643. The lipidomics analysis of this study was supported in part by the Lipidomics Shared Resource, Hollings Cancer Center, Medical University of South Carolina (P30 CA138313 and P30 GM103339). We thank the Department of Physiology (Chair Dr. Alan Daugherty) at the University of Kentucky, Lexington, KY for institutional support. 


\begin{abstract}
We have shown that deficiency of neutral sphingomyelinase 2 (nSMase2), an enzyme generating the sphingolipid ceramide, improves memory in adult mice. Here, we performed sphingolipid and RNA-seq analyses on the cortex from 10 month-old nSMase2-deficient (fro/fro) and heterozygous $(+/$ fro $)$ mice. frolfro cortex showed reduced levels of ceramide, particularly in astrocytes. Differentially abundant transcripts included several functionally related groups, with decreases in mitochondrial oxidative phosphorylation and astrocyte activation transcripts, while axon guidance and synaptic transmission transcripts were increased, indicating a role of nSMase 2 in oxidative stress, astrocyte activation, and cognition. Experimentally induced oxidative stress decreased the level of glutathione (GSH), an endogenous inhibitor of nSMase2, and increased immunolabeling for ceramide in primary $+/$ fro astrocytes, but not in fro/fro astrocytes. $\beta$-galactosidase activity was lower in 5-weeks old fro/fro astrocytes, indicating delayed senescence due to nSMase2 deficiency. In fro/fro cortex, levels of the senescence markers $\mathrm{C} 3 \mathrm{~b}$ and $\mathrm{p} 27$, and the proinflammatory cytokines interleukin $1 \beta$, interleukin 6 , and tumor necrosis factor $\alpha$ were reduced, concurrent with 2-fold decreased phosphorylation of their downstream target, protein kinase Stat3. RNA and protein levels of the ionotropic glutamate receptor subunit 2b (Grin2b or NR2B) were increased by 2-fold, an effect known to enhance cognition. This was consistent with 3.5 -fold reduced levels of exosomes carrying miR-223-3p, a micro-RNA downregulating Grin2b. In summary, our data show that nSMase2 deficiency prevents oxidative stress-induced elevation of ceramide and secretion of exosomes by astrocytes that suppress neuronal function, indicating a role of nSMase 2 in the regulation of neuroinflammation and cognition during brain aging.
\end{abstract}




\section{Significance statement}

Oxidative stress is associated with brain aging and cognitive decline. The underlying mechanism how oxidative stress impairs brain function is still not clear. We provide evidence that oxidative stress increases ceramide in astrocytes, which is prevented by deficiency of nSMase 2 , an enzyme that is activated by oxidative stress and generates ceramide from sphingomyelin. Mass spectrometric and transciptomic (RNA-seq) analyses show that in middle aged (10-month old) mouse cortex, nSMase2 deficiency reduces ceramide and increases expression of genes important for synaptic transmission and cognition. Therefore, our data show that oxidative stress-induced activation of nSMase 2 and generation of ceramide is significant for cognitive decline during aging. 


\section{Introduction}

The loss of cognition induced by the development of dementia represents one of the main pathological symptoms of human aging. Aging-related cognitive decline in humans can begin as early as middle age (45-65 years old) (Singh-Manoux et al., 2012). Cognitive decline related to the aging brain is associated with oxidative stress and neuroinflammation (Hajjar et al., 2018). Astrocytes, the most versatile cells in the central nervous system, play an important role in brain aging. The oxidative stress-preventing function of astrocytes is mainly due to their ability to generate endogenous anti-oxidants and regulate neuroinflammation (Lee et al., 2010). Most recently, it was shown that in reactive astrocytes, the level of the sphingolipid ceramide was increased (de Wit et al., 2019). Studies from our laboratory and others have shown that astrocytes increase the level of ceramide due to the activation of neutral sphingomyelinase 2 (nSMase2) by amyloid beta $(\mathrm{A} \beta)$ peptide and proinflammatory cytokines, the levels of both of which are increased during normal aging and in Alzheimer's disease (AD) (Singh et al., 1998; NikolovaKarakashian et al., 2008; Filippov et al., 2012; Wang et al., 2012; Gu et al., 2013; Dinkins et al., 2015; Dinkins et al., 2016; de Wit et al., 2019; Crivelli et al., 2020). We discovered that nSMase2 inhibition or deficiency ameliorated AD pathology in the 5XFAD mouse model, including cognitive improvement (Dinkins et al., 2014; Dinkins et al., 2016). Surprisingly, cognition was also improved in nSMase2-deficient mice without AD (Dinkins et al., 2016). These data suggested that downregulation of ceramide generation is beneficial for cognitive performance and prompted us to investigate the function of nSMase 2 and ceramide during normal aging of the brain. 
Activation of nSMase 2 and in turn, ceramide generation is induced by oxidative stress (NikolovaKarakashian et al., 2008). Oxidative stress is downregulated by astrocytes that are the main source for glutathione (GSH), an endogenous antioxidant, the level of which is strongly correlated with cognitive performance in the aging brain (Hajjar et al., 2018). The level of GSH determines the activation state of nSMase2 in hepatocytes (Liu and Hannun, 1997; Rutkute et al., 2007). Whether GSH also regulates nSMase2 in astrocytes is not known. If the GSH level drops below 5-8 mM, a concentration found in hepatocytes and astrocytes, nSMase2 is no longer inhibited and generates ceramide (Lee et al., 2010; McBean, 2017). In neurons, activation of nSMase2 by reduction of GSH is not likely since the neuronal GSH concentration $(<1 \mathrm{mM})$ is far below the range required for nSMase2 inhibition (McBean, 2017). Therefore, inhibition of nSMase2 by GSH is suggested to be critical for the regulation of ceramide levels in astrocytes, but not in neurons. Ceramide levels in nSMase2-deficient (fro/fro) astrocytes are expected to be insensitive to a decrease of GSH levels either as a result of aging or experimentally induced by oxidants.

In this study, we analyzed the ceramide and mRNA levels in the cortex of middle-aged (10 monthold) nSMase2-deficient (fro/fro) mice and control litermates (+/fro and/or wild type). In addition, we determined the effect of experimentally induced oxidative stress in vitro and analyzed proinflammatory cytokines and downstream cell signaling pathways activated by ceramide in vivo. Our studies show that nSMase 2 deficiency decreased ceramide levels and neuroinflammation, while levels of transcripts encoding proteins important for neuronal development and function were increased. Further, we show that nSMase2 deficiency reduces the level of exosomes transporting micro-RNAs that suppress expression of several/numerous genes important for 
bioRxiv preprint doi: https://doi.org/10.1101/2021.06.08.445892; this version posted June 9, 2021. The copyright holder for this preprint (which was not certified by peer review) is the author/funder. All rights reserved. No reuse allowed without permission.

neuronal function. These data indicate a critical role of nSMase 2 in the regulation of cognitive decline during brain aging. 


\section{Materials and Methods}

\section{Animals and reagents}

The fro/fro mouse was the gift from Dr. Christophe Poirier (Indiana University, Indianapolis). This carries a deletion of the C-terminal 33 amino acids in the sphingomyelin phosphodiesterase-3 (Smpd3) gene that encodes neutral sphingomyelinase 2 (nSMase2) (NCBI gene ID: 58994; (Aubin et al., 2005). Mice were handled according to the National Institutes of Health Guide for the Care and Use of Laboratory Animals. All procedures involving mice were approved by the Institutional Animal Care and Use Committee of University of Kentucky. Procedures focused on the use of male mice based on our previous studies showing that nSMase2 deficiency improves cognition in male 5XFAD mice (Dinkins et al., 2016). The antibodies, chemicals and reagents used in this study have been summarized and are listed in tables 2 and 3.

\section{Primary cell culture}

Primary astrocytes from fro/fro and littermate controls (wild type or heterozygous $+/$ fro) mice were prepared according to the protocol we have used previously (Dinkins et al., 2016; Kong et al., 2018). Astrocytes were maintained in DMEM with 10\% FBS and 1\% penicillin/streptomycin at $37{ }^{\circ} \mathrm{C}$ in a humidified atmosphere containing $5 \% \mathrm{CO}_{2}$. For all treatment procedures, including incubation with reagents and isolation of extracellular vesicles, astrocytes were first maintained for $48 \mathrm{~h}$ in serum-and phenol-red free DMEM medium.

\section{Immunoblotting}

Mouse brain cortex was solubilized by sonification in SDS-sample buffer containing 10\% 2mercaptoethanol and heated for $10 \mathrm{~min}$ at $95^{\circ} \mathrm{C}$ prior to SDS-PAGE and immunoblotting. In detail, 
proteins were resolved by SDS gel electrophoresis on polyacrylamide gels (Biorad, Hercules, California) and transferred to nitrocellulose membrane (Bio-Rad, Hercules, California). Nonspecific binding sites were blocked with 5\% fat-free dry milk (Bio-Rad Hercules, California) in PBS containing $0.1 \%$ Tween-20 (PBST) followed by overnight incubation with primary antibodies. The primary and secondary antibodies diluted with different concentrations in PBST are listed in the table 1 of antibodies and reagents. Signals were detected using either pico or femto chemiluminescent (ECL) horseradish peroxidase (HRP) substrate (Thermo Fisher, Massachusetts, USA). Blot images were captured by Azure c600 system (Azure Biosystems, California, USA).

\section{Immunocytochemistry and fluorescence microscopy}

Astrocytes grown on cover slips were fixed with 4\% p-formaldehyde/ $0.5 \%$ glutaraldehyde/PBS for $20 \mathrm{~min}$, followed by permeabilization with $0.2 \%$ Triton X-100 in PBS for 5-10 min at room temperature. Nonspecific binding sites were blocked with $3 \%$ ovalbumin/PBS for 1 hour at $37^{\circ} \mathrm{C}$. Cells were incubated with primary antibodies at $4{ }^{\circ} \mathrm{C}$ overnight. The next day, cells on cover slips were washed by PBS and followed by incubation with secondary antibodies for $2 \mathrm{~h}$ at room temperature. After washing with PBS, cover slips were mounted using Fluoroshield supplemented with DAPI (Sigma-Aldrich) to visualize the nuclei. Fluorescence microscopy was performed with a Nikon Ti2 Eclipse microscope equipped with NIS Elements software. Images were processed using a 3D deconvolution program as provided by the Elements software.

\section{Image analysis of colocalization studies}

A set of images was de-identified (blinded identifiers) and numbers assigned using a random generator program (random.org) available online. Colocalization was analyzed using the Nikon 
Elements software. The degree of colocalization was assessed by calculation of the Pearson's correlation coefficient for two fluorescence channels in overlays as previously described (Adler and Parmryd, 2010, 2013).

\section{Lipid analysis}

For ceramide analysis, brain tissues were submitted to the lipidomics core facility at the Medical University of South Carolina, Charlston, SC (Dr. Besim Ogretmen, director) (https://hollingscancercenter.musc.edu/). Quantitative analyses of sphingolipids were based on previous published methods (Bielawski et al., 2009, 2010). The concentration of ceramide species was quantified in the sphingolipidomics (LC-MS/MS) analysis core. The lipid concentration was normalized to lipid phosphate.

\section{RNA-seq}

RNA was extracted from the brain cortex using the miniRNeasy extraction kit (Qiagen). The total RNA was submitted to Novogene (https://en.novogene.com) for quality control and RNAseq analysis. Only the RNA with intergrity numbers $\geq 8$, and of sufficient purity (OD260/280=1.8-2.2; OD260/230 $\geq 1.8$ ) were used.

\section{Library preparation for transcriptome sequencing}

A total amount of 0.4 ug of RNA was used for cDNA library construction at Novogene using an NEBNext ${ }^{\circledR}$ Ultra 2 RNA Library Prep Kit for Illumina ${ }^{\circledR}$ (cat NEB \#E7775, New England Biolabs, Ipswich, MA, USA) according to the manufacturer's protocol. Briefly, mRNA was enriched using oligo(dT) beads. Double-stranded complementary DNA was synthesized, beginning with priming 
by random hexamers. After terminal repair, poly-adenylation, and sequencing adaptor ligation, the cDNA libraries were size-selected and enriched by PCR. The resulting 250-350 bp insert libraries were quantified using a Qubit 2.0 fluorometer (Thermo Fisher Scientific, Waltham, MA, USA) and quantitative PCR. Libraries were sequenced on an Illumina NovaSeq 6000 Platform (Illumina, San Diego, CA, USA) using a paired-end 150 run $(2 \times 150$ bases $)$. The number of raw reads per sample ranged from 50 to 60 Million. Paired-end reads were aligned to the mouse mm10 build reference genome using the Spliced Transcripts Alignment to a Reference (STAR) software. Identification of differentially abundant transcripts was done using DEseq2. The resulting P values were adjusted using the Benjamini and Hochberg approach for controlling the false discovery rate, and the threshold for differential expression.

\section{Functional Bioinformatics analysis}

The list of differentially abundant transcripts (fro/fro vs $+/$ fro) was imported to the Kyoto Encyclopedia of Genes and Genomes (KEGG) database to identify pathway annotations enriched among the proteins encoded by these transcripts. The lists of differentially abundant transcripts (fro/fro vs $+/$ fro) was also imported to the Ingenuity Pathway Analysis (IPA) software (Ingenuity H Systems, www.ingenuity.com)) to analyze functional annotations and regulatory networks via upstream regulator analysis, downstream effects analysis, mechanistic networks and causal network analysis.

\section{Exosome isolation and $q R T-P C R$}

Exosomes were isolated from $+/$ fro and fro/fro mice brain as described previously (Elsherbini et al., 2020b; Elsherbini et al., 2020a). miRNAs were eluted from exosomes using RNeasy minElute cleanup kit (Qiagen, 74204) and genomic DNA was digested with RNase-free DNase set (Qiagen, 
79254). miRNA reverse transcription was performed using miRCURY LNA reverse transcription kit (Qiagen, 339340). qRT-PCR was performed to amplify microRNA 223-3p using the primer hsa-miR-223-3p miRCURY LNA miRNA PCR Assay (Qiagen, \#339306, gene Globe ID: YP00205986) and LNA SYBR green PCR kit (Qiagen, 339345). Relative-fold changes were normalized comparing exosomal micro RNA reference gene miR-30c-5p using the primer hsamiR-30c-5p miRCURY LNA miRNA PCR Assay (Qiagen 339306, gene globe ID YP00204783). Relative fold changes of miR223-3p of fro/fro mice were calculated to $+/$ fro mice by using the

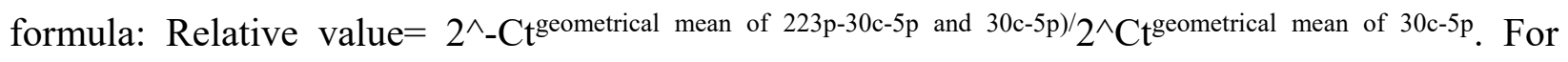
determination of the amount of exosomes in $+/$ fro and fro/fro mice, the right brain hemispheres of mice were digested in a mixture of papain (Sigma Aldrich, P3125) and hibernate A (Thermo Fisher Scientific, A1247501) at $37^{\circ} \mathrm{C}$ for $20 \mathrm{~min}$ and centrifuged at $300 \times g$ for $5 \mathrm{~min}$. Supernatants were centrifuged at $4000 \times g$ for $20 \mathrm{~min}$ and the resulting supernatants were recollected for further ultracentrifugation at $10,000 \times g$ for $40 \mathrm{~min}$. Finally, exosomes were collected by passing the supernatants through exoEasy columns (Qiagen, 76064) and quantification was performed by nanoparticle tracking analysis (NTA) with ZetaView PMX110 (Particle Metrix).

\section{Statistical Analysis}

Statistical analyses and graphing were performed using Microsoft Excel 2019 and GraphPad Prism 8.0 software (GraphPad, San Diego, CA, USA). For IPA analyses, a $Z$ score $(Z \leq-2.0$ or $Z \geq 2.0)$ was considered significant. When the two groups were compared, student $t$ test was used. Values of $P<0.05$ were considered significant. When multiple groups $(>2)$ were compared, two-way ANOVA was applied. 
Table 1. List of Antibodies used in the study

\begin{tabular}{llll}
\hline Antibodies & Ratio & Cat\# & Company \\
\hline Anti-IL-1 $\beta$ & $1: 250$ & SC-32294 & Santa Cruz \\
Anti-IL-6 & $1: 250$ & SC-32296 & Santa Cruz \\
Anti-TNF $\alpha$ & $1: 250$ & SC-12744 & Santa Cruz \\
Anti-NR2B & $1: 1000$ & $06-600$ & Millipore Sigma \\
Anti-Stat3 & $1: 1000$ & $\# 4904$ & Cell Signaling \\
Anti-pStat3 & $1: 1000$ & $\# 9145$ & Cell Signaling \\
Anti-P27 kip1 & $1: 1000$ & \#2552 & Cell Signaling \\
Anti-ceramide rabbit IgG & $1: 100$ & Were generated in our laboratory \\
Anti-ceramide mouse IgM & $1: 100$ & MAS0014 & Glycobiotech \\
Anti-rabbit HRP & $1: 5000$ & $715-035-152$ & Jackson Immune Research \\
Anti-mouse HRP & $1: 10,000$ & 20071831 & DaKo \\
Anti-GAPDH & $1: 5000$ & $715-035-151$ & Jackson Immune Research \\
\hline
\end{tabular}


Table 2. Chemicals and Kits used in the study

\begin{tabular}{|c|c|c|}
\hline Reagents & Cat\# & Company \\
\hline SDS-PAGE sample buffer & S3401-1VL & Sigma-Aldrich \\
\hline Protease and phosphatase inhibitors & A32961 & ThermoFisher Scientific \\
\hline Fetal Bovine Serum & E19057 & Atlanta Biologicals \\
\hline DMEM & 2261905 & Hyclon \\
\hline Penicillin and streptomycin & 15146 & GIBCO \\
\hline GW4869 & $6823-69-4$ & Cayman \\
\hline $\mathrm{tBO} 2 \mathrm{H}$ & 458139 & Sigma-Aldrich \\
\hline N-acetyl-L-Cysteine (NAcCys) & 20261 & Cayman \\
\hline$\beta$-galactosisdase & \#9860 & Cell signaling tech \\
\hline Monochlorobimane (MCB) & 69899 & Sigma-Aldrich \\
\hline RNeasy plus mini kit & 74134 & QIAGEN \\
\hline RNeasy minElute cleanup kit & 74204 & QIAGEN \\
\hline RNase-Free DNase set & 79254 & QIAGEN \\
\hline miRCURY LNA reverse transcription kit & 339340 & QIAGEN \\
\hline primer hsa-miR-223-3p miRCURY LNA & 339306 & QIAGEN \\
\hline \multicolumn{3}{|l|}{ miRNA PCR Assay } \\
\hline LNA SYBR green PCR kit & 339345 & QIAGEN \\
\hline exoEasy column & 76064 & QIAGEN \\
\hline Hibernate A & A1247501 & Thermo Fisher Scientific \\
\hline papain & P3125 & Sigma Aldrich \\
\hline
\end{tabular}




\section{Results}

\section{nSMase2 deficiency prevents age-related increase of ceramide levels in astrocytes}

To determine if nSMase 2 deficiency affects ceramide levels in the middle-aged brain, the cortex of 10-month old fro/fro and +/fro (heterozygous litermates) were subjected to targeted liquid chromatography-tandem mass spectrometry (LC-MS/MS). Figure 1 shows that the levels of all ceramide species were decreased in fro/fro cortex, with the ceramide species $\mathrm{C}_{18: 0}, \mathrm{C}_{18: 1}$, and $\mathrm{C}_{24: 1}$ ceramide being downregulated the most. It was previously shown, that these ceramide species were upregulated in the aging brain and serum, and associated with memory impairment, including AD (Cutler et al., 2004; Haughey et al., 2010; Mielke et al., 2010a; Mielke et al., 2010b; Mielke and Lyketsos, 2010; Mielke et al., 2012).

Using immunocytochemistry with an anti-ceramide antibody developed in our laboratory (Krishnamurthy et al., 2007), we tested if ceramide levels were affected depending on age and cell type. Figure 2 shows that labeling for ceramide was increased up to 4-fold in 10 and 15 month-old +/fro mice vs. 3 month-old mice, and in +/fro brains (from 15 month-old mice) when compared to 15 month-old fro/fro tissue. More importantly, increased ceramide labeling was predominantly colocalized with GFAP positive processes in +/fro tissue (arrows), while labeling in neurons was >2-times lower and confined to perinuclear compartments. In 10 and 15 month-old mice, punctate ceramide labeling was often juxtaposed to astrocytes indicating a secretory process (arrows in Fig. 2B-E). Ceramide labeling of microglia (Iba-1 positive) was not increased during brain aging and it was not altered by nSMase 2 deficiency. These data suggest that nSMase 2 deficiency mainly prevented elevation of ceramide and its secretion by astrocytes in the middle-aged brain. 


\section{nSMase2 deficiency alters $m R N A$ abundance in the middle-aged brain}

We performed RNAseq analyses on +/fro and fro/fro cortex to screen for clues as to how the lack of ceramide generation due to nSMase 2 deficiency affects the middle-aged brain. A total of 1462 transcripts were changed, among them, 891 transcripts were increased and 571 transcripts were decreased. The most increased transcript was Grin2b (arrow in Fig. 3A). The differences in transcript abundance observed did not include differences in markers of specific cell types, and therefore did not argue for differences in the proportions of types of neurons or glia between the genotypes tested. This observation argues that these transcript abundance differences arose from differences in gene expression.

Functional bioinformatics analyses revealed that synaptic signaling annotations were overrepresented among the transcripts that were increased in the nSMase2-deficient cortex and many of the encoded proteins, such as Grin2b, are important for memory (Fig. 3B and C). These analyses also revealed that nSMase 2 deficiency was associated with increased levels of mRNAs encoding proteins involved in axonal guidance and long-term potentiation, suggesting that neural plasticity may be enhanced. Furthermore, these analyses revealed that annotations for neurodegenerative conditions such as AD, Parkinson's disease, and Huntington's disease were over-represented among the transcripts that decreased in fro/fro mouse brains (Fig. 3D). According to the KEGG analysis, also decreased was a set of transcripts encoding mitochondrial complex proteins important for oxidative phosphorylation, indicating that nSMase 2 is involved in regulation of mitochondrial oxidative stress. (Fig. 3E). These discoveries are consistent with enhanced memory function and increased neural protection, and therefore provide the likely mechanistic explanations for the enhanced memory outcomes observed in fro/fro mice (Dinkins et al., 2016). 
nSMase2 deficiency or inhibition prevents oxidative stress-induced increase of ceramide levels and activation of astrocytes

In astrocytes, oxidative phosphorylation in mitochondria is reduced in favor of aerobic glycolysis, which was suggested to diminish oxidative stress and protect neurons (Demetrius and Simon, 2012; Tavallaie et al., 2020; Zheng et al., 2021). Oxidative stress, however, is increased in reactive astrocytes and induces the production of proinflammatory cytokines (Lee et al., 2010). Based on the KEGG pathway enrichment analysis showing that a set of transcripts encoding proteins participating in mitochondrial oxidative phosphorylation were decreased in fro/fro brain (Fig. 3D and E), we hypothesized that nSMase2 deficiency decreased astrocyte activation, which was consistent with reduced levels of transcripts for S100 3 and C1q, two markers for activated astrocytes (not shown).

Since astrocyte activation has been associated with increased oxidative stress and activation of nSMase2, we tested the response of primary cultured heterozygous $(+/$ fro $)$ and fro/fro astrocytes to oxidative stress induced by incubation with tertiary butyl peroxide $(\mathrm{tBO} 2 \mathrm{H})$. We used monochlorobimane (MCB), a fluorescence indicator for glutathione (GSH), and the anti-ceramide antibody to detect the level of GSH and ceramide in tBO2H-treated astrocytes using immunofluorescence microscopy. Figure 4A shows that incubation of astrocytes with tBO2H led to the reduction of MCB fluorescence in both, +/fro and fro/fro astrocytes, while ceramide labeling was only increased in +/fro astrocytes. This result indicated that oxidant-induced loss of GSH activated nSMase 2 and led to the generation of ceramide in heterozygous astrocytes, which was absent in fro/fro astrocytes. Likewise, GFAP labeling, as a marker of activated astrocytes, was only increased in heterozygous cells, indicating that $\mathrm{tBO} 2 \mathrm{H}$ incubation led to activation of 
astrocytes, which required activation of nSMase 2 by oxidative stress. This conclusion was further supported by colocalization analyses showing that in control (non-treated) as well as tBO2Htreated heterozygous astrocytes, loss of MCB labeling was associated with strong ceramide labeling, and that the increase of ceramide labeling was directly correlated with that of GFAP (Pearson's colocalization coefficient $0.65 \pm 0.15$ ).

To test if restoration of GSH levels prevented oxidative-stress induced activation of nSMase 2 and generation of ceramide, we pre-incubated tBO2H-treated wild type astrocytes with $\mathrm{N}$-acetyl cysteine (NAcCys), an endogenous antioxidant known to provide cysteine to boost GSH production and reduce oxidative stress in vitro and in vivo (Farr et al., 2003; Rutkute et al., 2007; Cao et al., 2012; Skvarc et al., 2017). Figure 4B shows that NAcCys (NAc) prevented depletion of GSH and increase of ceramide labeling. GW4869, an inhibitor of nSMase2, did not block $\mathrm{tBO} 2 \mathrm{H}$-induced GSH depletion, but prevented the increase of ceramide in the presence of $\mathrm{tBO} 2 \mathrm{H}$, confirming that onset of oxidative stress is upstream of nSMase-2 activation and generation of ceramide. Taken together, these results suggest that oxidative stress leads to the decrease of GSH levels and nSMase2-mediated generation of ceramide.

\section{nSMase2 deficiency reduces astrocyte senescence and inflammation in the brain}

Oxidative stress-induced astrocyte activation has been associated with inflammation and astrocyte senescence in the aging brain (Lee et al., 2010). Further, the onset of senescence in astrocytes is an essential part of the mechanisms underlying functional decline during brain aging (Cohen and Torres, 2019). Since both, oxidative stress and ceramide have been implicated in cellular senescence, we tested if nSMase 2 deficiency delayed senescence of astrocytes. We assayed the 
activity of a senescence marker, $\beta$-galactosidase $(\beta$-gal), in 5-weeks old primary cultures of $+/$ fro vs. fro/fro astrocytes. Figure 5A and B shows that $\beta$-gal staining was lower in nSMase2-deficient astrocytes (fro/fro) as compared to +/fro astrocytes, even after normalizing for cell density suggesting that lack of nSMase 2 delayed aging of astrocytes. $\beta$-gal staining in aging $+/$ fro astrocytes was similar to that in wild type astrocytes, indicating that a single allele of nSMase 2 is sufficient for ceramide generation underlying astrocyte senescence (not shown).

Based on the results of the $\beta$-gal in vitro assay, suggesting that senescence was decreased in nSMase2-deficient astrocytes, we determined the level of senescence markers in fro/fro brain tissue. Consistent with the in vitro data in primary astrocytes, Figure $5 \mathrm{C}$ shows that the levels of two senescence markers, $\mathrm{p} 27$ and C3b were lower in the middle-aged fro/fro brain as compared to the wild type control. Since senescence in the brain is associated with neuroinflammation, we next determined the level of proinflammatory cytokines using immunoblot analysis. Figure 5D shows that IL-1 $\beta$ and IL-6, were significantly decreased in fro/fro mouse brain, suggesting that nSMase 2 deficiency reduces inflammation in the middle-aged brain.

Upregulation of proinflammatory or senescence markers is characteristic of reactive astrocytes (Escartin et al., 2021). Proinflammatory cytokines induce tyrosine (Y705) phosphorylation of Stat3, a transcription factor activated in reactive and aging astrocytes (Herrmann et al., 2008; Hashioka et al., 2011; O'Callaghan et al., 2014; White et al., 2020). Consistent with decreased levels of proinflammatory cytokines, we observed significantly reduced phosphorylation of Stat3 in fro/fro brains as compared to wild type (Fig. 5E). This indicates that nSMase2 participates in 
the upregulation of inflammation in the middle-aged brain through activation of the Stat 3 signaling pathway.

\section{nSMase2 deficiency increases axonal growth and glutamate signaling transcripts}

In contrast to the suppressive effect of nSMase 2 deficiency on several transcripts encoding proteins important in astrocyte activation, senescence, and neuroinflammation, our data clearly show increases in sets of transcripts encoding proteins involved in axonal guidance/growth and neural signal transmission, especially synaptic signaling and plasticity related to cognition, memory, and learning (Table 3). Figure 6A displays the primary network identified when the whole set of differentially abundant transcripts was analyzed by IPA. The sub-networks include motor-function related coordination, processing of RNA and several transcription regulation networks, such as those driven by MYC and MYCN. The motor-related behavioral coordination networks involve Grin2b, Grin2a, and BDNF. BDNF is predicted to positively regulate Grin2b in coordinationassociated networks (Fig. 6A, B) (Zhang et al., 2018). More importantly, Grin2b is a critical component of the N-methyl-D-aspartate receptors that mediate forms of long-term potentiation fundamental to cognitive functions and memory (Fig. 7A) (Gozlan et al., 1995). To confirm that upregulation of Grin $2 \mathrm{~b}$ transcript results in increased amount of protein, we determined the protein levels of Grin2b in the brain cortex of both wild type and fro/fro mice (10-month old). Consistent with the RNAseq data, we found that the protein level of Grin2b in the brain cortex was two-times higher in fro/fro mice than in wild type mice (Fig. 7B).

Increases in Grin2b mRNA may be induced by nSMase2 deficiency in neurons or other cell types, such as astrocytes. Since immunolabeling for ceramide was increased in astrocytes of the aging 
brain, we investigated a potential trans-cellular effect of astrocytes on gene expression in neurons. Two potential factors suppressing expression of Grin2b are the neural differentiation transcription factor REST and the micro-RNA miR-223-3p (Harraz et al., 2012; Rodenas-Ruano et al., 2012) (Fig. 8A). We previously published that reactive astrocytes secrete exosomes enriched with ceramide, consistent with punctate labeling for ceramide in juxtaposition to astrocytic processes in 10 and 15-month old mouse cortex (Fig. 2) (Wang et al., 2012). A recent study showed that exosomes secreted by reactive astrocytes were enriched with miR-223-3p and downregulated Grin2b mRNA when added to neuronal cultures (Amoah et al., 2020). To test if nSMase2 regulated the amount of exosomal miR-223-3p, we performed qRT-PCR for miR-223-3p in brain derivedexosomes from 10-month old +/fro and fro/fro mice. This experiment did not reveal a significant decrease when compared to exosomal housekeeping micro-RNA (Fig. 8B), but it also did not rule out contributions from small changes in miR-223-3p to the increased amounts of Grin2b in fro/fro mice. More importantly, quantitation of exosomes showed that their number was reduced by $70 \%$ in the fro/fro cortex when compared to that of $+/$ fro mice (Fig. $8 \mathrm{C}$ and D). Therefore, even if the copy number/exosomes of miR-223-3p is not significantly reduced, the decrease of exosomes secretion due to nSMase2 deficiency will lower the impact of this micro-RNA on the amount of Grin2b in fro/fro mice.

\section{Discussion}

We have previously shown that nSMase 2 deficiency (fro/fro mouse) led to improved cognition in 5XFAD mice (Dinkins et al., 2016). During these studies, we detected marked improvement of memory function (fear conditioning test) in non-AD fro/fro controls when compared to wild type littermates, suggesting that reduced cognitive decline due to nSMase 2 deficiency involves mechanisms independent of $\mathrm{AD}$. To identify these mechanisms regulated by nSMase2, we 
performed transcriptomic (RNAseq) analyses on cortices of homozygous (fro/fro) and heterozygous (+/fro) mice. We focused on middle-aged male mice, since our studies on AD showed that cognitive improvement due to nSMase2 deficiency was limited to middle-aged males.

Consistent with improved cognition, RNA-seq analysis revealed increases in clusters of transcripts encoding axonal guidance and synaptic signal transmission proteins in the cortex of fro/fro mice (Table 3, Fig. 3). The coordinated increase in these sets of functionally related transcripts due to nSMase2 deficiency suggests that nSMase2 regulates neuronal function either intrinsically or by trans-cellular effects arising from other cell types, particularly astrocytes. Evidence against intrinsic effects arises from decreases in transcripts encoding mitochondrial proteins important for oxidative phosphorylation in fro/fro cortex (Fig. 3). Oxidative phosphorylation is critical for neurons, but not for astrocytes, which mainly generate ATP from aerobic glycolysis (Demetrius and Simon, 2012; Zheng et al., 2021). Hence, if our evidence for decreased capacity for oxidative phosphorylation stems from downregulation of oxidative phosphorylation in neurons this would impede enhanced synaptic transmission and cognitive performance. In addition, recent studies show that downregulation of oxidative phosphorylation and upregulation of aerobic glycolysis in astrocytes is protective for neurons and ameliorates AD pathology (Demetrius and Simon, 2012; Zheng et al., 2021). Therefore, the current study is based on the hypothesis that decreases in transcripts encoding proteins involved in oxidative phosphorylation are limited to astrocytes. Indeed, the results are consistent with this hypothesis showing reduction of glial activation and neuroinflammation as indicated by decreased levels of S100 $\beta$ and C1q transcripts and proinflammatory cytokines (Fig. 3 and 5D), as well as reduced astrocyte senescence (Fig. 5A and B). 
Increased astrocyte activation and neuroinflammation are hallmarks of the aging brain and associated with reduced levels of GSH, an endogenous antioxidant mainly produced in astrocytes. There is a strong correlation between cognitive decline and lower levels of GSH (Hajjar et al., 2018). The GSH levels in the brain decrease with age, mainly by being used to neutralize reactive oxygen species (ROS) (Zhu et al., 2006). Astrocytes are the main source for the GSH precursor dipeptide (Cys-Gly) used for GSH biosynthesis in neurons (McBean, 2017). Astrocytes maintain their neuroprotective role under acute oxidative stress (Bhatia et al., 2019), but they become depleted of GSH during aging and neurodegenerative disease (Lee et al., 2010). The immediate target affected by GSH depletion in astrocytes is still unknown. Our in vitro experiments with primary cultured astrocytes show that reduction of GSH due to oxidative stress induces generation of ceramide, which is prevented by inhibition or deficiency of nSMase2 (Fig. 4). It is known that the GSH concentration found in astrocytes $(5-8 \mathrm{mM})$ is in the range required for inhibition of nSMase2 (Liu and Hannun, 1997; Liu et al., 1998; Rutkute et al., 2007; Nikolova-Karakashian et al., 2008; Lee et al., 2010; McBean, 2017). Hence, oxidative stress leading to GSH depletion will activate nSMase 2 and in turn, induce generation of ceramide.

Lipidomics analysis shows that in the aging brain, all major ceramide species, including those found to be predominantly reduced in nSMase2-deficient fro/fro brain $\left(\mathrm{C}_{18: 0}, \mathrm{C}_{18: 1}\right.$, and $\mathrm{C}_{24: 1}$ ceramides) are elevated (Cutler et al., 2004; Haughey et al., 2010; Mielke et al., 2010a; Mielke et al., 2010b; Mielke and Lyketsos, 2010; Mielke et al., 2012). Since the reduction of GSH levels in the aging brain is strongly associated with cognitive decline, we explored the hypothesis that the decrease of GSH levels leads to the activation of nSMase 2 and generation of ceramide during aging. The strongest driver of GSH depletion during aging is oxidative stress leading to reactive 
oxygen metabolites that deplete GSH. Therefore, it is reasonable to conclude that the in vitro experiments showing increased generation of ceramide by oxidative stress-induced activation of nSMase 2 in astrocytes recapitulate a mechanism similar to that in the aging brain.

Previous studies have shown that oxdative stress activates astrocytes and leads to the secretion of proinflammatory cytokines (Escartin et al., 2021). Since these cytokines induce activation of nSMase2 and ceramide participates in mediating signaling downstream of cytokine receptors, we tested if neuroinflammation is reduced in nSMase2-deficient brain. The immunoblot data on inflammation and senescence markers showed that fro/fro mice cortex contained decreased levels of the proinflammatory cytokines IL-1 $\beta$, IL- 6 , and TNF- $\alpha$, and lower levels of the senescence markers p27 and C3b (Fig. 5C and D). These data are consistent with a lower phosphorylation level of Stat3 (Y705), the major protein kinase activated by proinflammatory cytokines, upregulated during aging, and characteristic for reactive astrocytes (Fig. 5E) (Herrmann et al., 2008; Hashioka et al., 2011; O'Callaghan et al., 2014; White et al., 2020). Downregulation of the Stat3-associated cell signaling pathways suggests that aging-related processes, particularly activation and senescence of astrocytes are prevented or delayed in nSMase2-deficient fro/fro brain. This result is consistent with our in vitro data on nSMase2-deficient astrocytes, which age slower than wild type astrocytes as shown by $\beta$-galactosidase staining in 5 -week old primary cultures of fro/fro vs. +/fro astrocytes (Fig. 5A and B).

In agreement with delayed brain aging, nSMase 2 deficiency is associated with sets of transcripts that encode proteins involved in axon development and synaptic signal transmission (Fig. 3). Particularly, the mRNA and protein levels of Grin2b, the b-subunit of the ionotropic glutamate 
receptor are increased by about 2-fold in fro/fro cortex (Figs. 3A and 7B). The level of Grin2b upregulation is similar to that of a transgenic mouse generated about 20 years ago (Tang et al., 1999; Cao et al., 2007). The aged Grin2b overexpressing mice outperformed their wild-type littermates in five different learning and memory behavior tests, including fear conditioning tests. In fact, the performance of these mice in the fear conditioning test was very similar to that of the fro/fro mouse, consistent with a similar expression level of Grin2b. Conversely, the subunit composition of the NMDA receptor shows a significant decrease in NR1, NR2A, and NR2B (Grin2b) at the synaptic protein level during aging and in AD (Avila et al., 2017). This raises the interesting possibility that downregulation of the NMDA receptor, especially the Grin2b subunit, contributes to decreased cognitive function during the aging process. Therefore, the increased level of Grin2b in fro/fro mice suggests that nSMase 2 deficiency is beneficial for learning and memory function in the middle-aged brain.

Since nSMase2 deficiency decreases the level of ceramide, it is critical to understand how and in which cell type lack of nSMase 2 activity prevents ceramide-induced aging of the brain and cognitive decline. Since nSMase 2 deficiency is not confined to astrocytes, reduction of ceramide levels may be critical in neurons and other cells as well. Immunolabeling for ceramide in cryosections shows that the level of ceramide is mainly increased in astrocytes of the aging brain, and lower in astrocytic processes of fro/fro brain (Fig. 2). In addition, punctate labeling of ceramide in close vicinity to GFAP $(+)$ astrocytic processes indicates ceramide secretion, which is known for reactive astrocytes that secrete ceramide-enriched exosomes or "astrosomes" (Wang et al., 2012). These data suggest that aging-related increase of ceramide in wild type astrocytes may have a trans-cellular effect on other cells such as neurons via secretion of exosomes. 
Recently, it was shown that activated astrocytes secrete exosomes that are enriched with miR-2233p, a micro-RNA that downregulates Grin2b levels in neurons (Fig. 8A) (Amoah et al., 2020). Our data show reduced levels of exosomes in fro/fro brain, including those carrying miR-223-3p (Fig. 8B-D). These data are consistent with our previous studies showing that nSMase 2 deficiency or inhibition reduced exosome secretion from in vitro-cultivated astrocytes and brain exosome levels (Wang et al., 2012; Dinkins et al., 2016). Therefore, it is possible that nSMase2 deficiency reduces exosomal micro-RNAs that downregulate neuronal function, which could explain the increase of the respective mRNAs levels in fro/fro brain.

One may speculate that GSH and ceramide metabolism are intertwined in astrocytes and neurons of the aging brain (Fig. 9). In astrocytes, oxidative phosphorylation is downregulated in favor of aerobic glycolysis, which produces reduction equivalents (NADH) to regenerate GSH. GSH inhibits nSMase2-mediated ceramide generation and secretion of astrosomes. Increased oxidative stress during aging leads to reduction of GSH levels in astrocytes and activation of nSMase2, which induces formation and secretion of aging-associated astrosomes (AAAs) that suppress neuronal activity. In nSMase2-deficient (fro/fro) mice, lack of ceramide elevation prevents formation of AAAs, and in turn, preserves neuronal function. Hence, GSH as a sensor for oxidative stress and nSMase2-catalyzed generation of ceramide may constitute a feedback loop for the function of AAAs in the regulation of neuronal activity. In our future studies, we will further investigate the role of nSMase 2 and ceramide in oxidative stress and aging-related regulation of neuronal activity and cognition. 


\section{References}

Adler J, Parmryd I (2010) Quantifying colocalization by correlation: the Pearson correlation coefficient is superior to the Mander's overlap coefficient. Cytometry A 77:733-742.

Adler J, Parmryd I (2013) Colocalization analysis in fluorescence microscopy. Methods Mol Biol 931:97-109.

Amoah SK, Rodriguez BA, Logothetis CN, Chander P, Sellgren CM, Weick JP, Sheridan SD, Jantzie LL, Webster MJ, Mellios N (2020) Exosomal secretion of a psychosis-altered miRNA that regulates glutamate receptor expression is affected by antipsychotics. Neuropsychopharmacology : official publication of the American College of Neuropsychopharmacology 45:656-665.

Aubin I, Adams CP, Opsahl S, Septier D, Bishop CE, Auge N, Salvayre R, Negre-Salvayre A, Goldberg M, Guenet JL, Poirier C (2005) A deletion in the gene encoding sphingomyelin phosphodiesterase $3(\mathrm{Smpd} 3)$ results in osteogenesis and dentinogenesis imperfecta in the mouse. Nat Genet 37:803-805.

Avila J, Llorens-Martin M, Pallas-Bazarra N, Bolos M, Perea JR, Rodriguez-Matellan A, Hernandez F (2017) Cognitive Decline in Neuronal Aging and Alzheimer's Disease: Role of NMDA Receptors and Associated Proteins. Frontiers in neuroscience 11:626.

Bhatia TN, Pant DB, Eckhoff EA, Gongaware RN, Do T, Hutchison DF, Gleixner AM, Leak RK (2019) Astrocytes Do Not Forfeit Their Neuroprotective Roles After Surviving Intense Oxidative Stress. Front Mol Neurosci 12:87.

Bielawski J, Pierce JS, Snider J, Rembiesa B, Szulc ZM, Bielawska A (2009) Comprehensive quantitative analysis of bioactive sphingolipids by high-performance liquid chromatography-tandem mass spectrometry. Methods Mol Biol 579:443-467. 
Bielawski J, Pierce JS, Snider J, Rembiesa B, Szulc ZM, Bielawska A (2010) Sphingolipid analysis by high performance liquid chromatography-tandem mass spectrometry (HPLC-MS/MS). Adv Exp Med Biol 688:46-59.

Cao L, Li L, Zuo Z (2012) N-acetylcysteine reverses existing cognitive impairment and increased oxidative stress in glutamate transporter type 3 deficient mice. Neuroscience 220:85-89.

Cao X, Cui Z, Feng R, Tang YP, Qin Z, Mei B, Tsien JZ (2007) Maintenance of superior learning and memory function in NR2B transgenic mice during ageing. Eur J Neurosci 25:18151822.

Cohen J, Torres C (2019) Astrocyte senescence: Evidence and significance. Aging Cell 18:e12937. Crivelli SM, Giovagnoni C, Visseren L, Scheithauer AL, de Wit N, den Hoedt S, Losen M, Mulder MT, Walter J, de Vries HE, Bieberich E, Martinez-Martinez P (2020) Sphingolipids in Alzheimer's disease, how can we target them? Advanced drug delivery reviews.

Cutler RG, Kelly J, Storie K, Pedersen WA, Tammara A, Hatanpaa K, Troncoso JC, Mattson MP (2004) Involvement of oxidative stress-induced abnormalities in ceramide and cholesterol metabolism in brain aging and Alzheimer's disease. Proc Natl Acad Sci U S A 101:20702075.

de Wit NM, den Hoedt S, Martinez-Martinez P, Rozemuller AJ, Mulder MT, de Vries HE (2019) Astrocytic ceramide as possible indicator of neuroinflammation. Journal of neuroinflammation 16:48.

Demetrius LA, Simon DK (2012) An inverse-Warburg effect and the origin of Alzheimer's disease. Biogerontology 13:583-594. 
Dinkins MB, Dasgupta S, Wang G, Zhu G, Bieberich E (2014) Exosome reduction in vivo is associated with lower amyloid plaque load in the 5XFAD mouse model of Alzheimer's disease. Neurobiol Aging 35:1792-1800.

Dinkins MB, Dasgupta S, Wang G, Zhu G, He Q, Kong JN, Bieberich E (2015) The 5XFAD Mouse Model of Alzheimer's Disease Exhibits an Age-Dependent Increase in AntiCeramide IgG and Exogenous Administration of Ceramide Further Increases AntiCeramide Titers and Amyloid Plaque Burden. J Alzheimers Dis.

Dinkins MB, Enasko J, Hernandez C, Wang G, Kong J, Helwa I, Liu Y, Terry AV, Jr., Bieberich E (2016) Neutral Sphingomyelinase-2 Deficiency Ameliorates Alzheimer's Disease Pathology and Improves Cognition in the 5XFAD Mouse. J Neurosci 36:8653-8667.

Elsherbini A, Qin H, Zhu Z, Tripathi P, Crivelli SM, Bieberich E (2020a) In vivo evidence of exosome-mediated Abeta neurotoxicity. Acta neuropathologica communications 8:100.

Elsherbini A, Kirov AS, Dinkins MB, Wang G, Qin H, Zhu Z, Tripathi P, Crivelli SM, Bieberich E (2020b) Association of Abeta with ceramide-enriched astrosomes mediates Abeta neurotoxicity. Acta Neuropathol Commun 8:60.

Escartin C et al. (2021) Reactive astrocyte nomenclature, definitions, and future directions. Nature neuroscience 24:312-325.

Farr SA, Poon HF, Dogrukol-Ak D, Drake J, Banks WA, Eyerman E, Butterfield DA, Morley JE (2003) The antioxidants alpha-lipoic acid and N-acetylcysteine reverse memory impairment and brain oxidative stress in aged SAMP8 mice. J Neurochem 84:1173-1183.

Filippov V, Song MA, Zhang K, Vinters HV, Tung S, Kirsch WM, Yang J, Duerksen-Hughes PJ (2012) Increased ceramide in brains with Alzheimer's and other neurodegenerative diseases. J Alzheimers Dis 29:537-547. 
Gozlan H, Khazipov R, Ben-Ari Y (1995) Multiple forms of long-term potentiation and multiple regulatory sites of N-methyl-D-aspartate receptors: role of the redox site. J Neurobiol $26: 360-369$.

Gu L, Huang B, Shen W, Gao L, Ding Z, Wu H, Guo J (2013) Early activation of nSMase2/ceramide pathway in astrocytes is involved in ischemia-associated neuronal damage via inflammation in rat hippocampi. J Neuroinflammation 10:109.

Hajjar I, Hayek SS, Goldstein FC, Martin G, Jones DP, Quyyumi A (2018) Oxidative stress predicts cognitive decline with aging in healthy adults: an observational study. J Neuroinflammation 15:17.

Harraz MM, Eacker SM, Wang X, Dawson TM, Dawson VL (2012) MicroRNA-223 is neuroprotective by targeting glutamate receptors. Proceedings of the National Academy of Sciences of the United States of America 109:18962-18967.

Hashioka S, Klegeris A, Qing H, McGeer PL (2011) STAT3 inhibitors attenuate interferongamma-induced neurotoxicity and inflammatory molecule production by human astrocytes. Neurobiology of disease 41:299-307.

Haughey NJ, Bandaru VV, Bae M, Mattson MP (2010) Roles for dysfunctional sphingolipid metabolism in Alzheimer's disease neuropathogenesis. Biochim Biophys Acta 1801:878886.

Herrmann JE, Imura T, Song B, Qi J, Ao Y, Nguyen TK, Korsak RA, Takeda K, Akira S, Sofroniew MV (2008) STAT3 is a critical regulator of astrogliosis and scar formation after spinal cord injury. The Journal of neuroscience : the official journal of the Society for Neuroscience 28:7231-7243. 
Kong JN, Zhu Z, Itokazu Y, Wang G, Dinkins MB, Zhong L, Lin HP, Elsherbini A, Leanhart S, Jiang X, Qin H, Zhi W, Spassieva SD, Bieberich E (2018) Novel function of ceramide for regulation of mitochondrial ATP release in astrocytes. J Lipid Res 59:488-506.

Krishnamurthy K, Dasgupta S, Bieberich E (2007) Development and characterization of a novel anti-ceramide antibody. J Lipid Res 48:968-975.

Lee M, Cho T, Jantaratnotai N, Wang YT, McGeer E, McGeer PL (2010) Depletion of GSH in glial cells induces neurotoxicity: relevance to aging and degenerative neurological diseases. FASEB journal : official publication of the Federation of American Societies for Experimental Biology 24:2533-2545.

Liu B, Hannun YA (1997) Inhibition of the neutral magnesium-dependent sphingomyelinase by glutathione. J Biol Chem 272:16281-16287.

Liu B, Andrieu-Abadie N, Levade T, Zhang P, Obeid LM, Hannun YA (1998) Glutathione regulation of neutral sphingomyelinase in tumor necrosis factor-alpha-induced cell death. J Biol Chem 273:11313-11320.

McBean GJ (2017) Cysteine, Glutathione, and Thiol Redox Balance in Astrocytes. Antioxidants (Basel) 6 .

Mielke MM, Lyketsos CG (2010) Alterations of the sphingolipid pathway in Alzheimer's disease: new biomarkers and treatment targets? Neuromolecular Med 12:331-340.

Mielke MM, Bandaru VV, Haughey NJ, Rabins PV, Lyketsos CG, Carlson MC (2010a) Serum sphingomyelins and ceramides are early predictors of memory impairment. Neurobiology of aging 31:17-24.

Mielke MM, Haughey NJ, Bandaru VV, Schech S, Carrick R, Carlson MC, Mori S, Miller MI, Ceritoglu C, Brown T, Albert M, Lyketsos CG (2010b) Plasma ceramides are altered in 
mild cognitive impairment and predict cognitive decline and hippocampal volume loss. Alzheimers Dement 6:378-385.

Mielke MM, Bandaru VV, Haughey NJ, Xia J, Fried LP, Yasar S, Albert M, Varma V, Harris G, Schneider EB, Rabins PV, Bandeen-Roche K, Lyketsos CG, Carlson MC (2012) Serum ceramides increase the risk of Alzheimer disease: the Women's Health and Aging Study II. Neurology 79:633-641.

Nikolova-Karakashian M, Karakashian A, Rutkute K (2008) Role of neutral sphingomyelinases in aging and inflammation. Subcell Biochem 49:469-486.

O'Callaghan JP, Kelly KA, VanGilder RL, Sofroniew MV, Miller DB (2014) Early activation of STAT3 regulates reactive astrogliosis induced by diverse forms of neurotoxicity. PloS one 9:e102003.

Rodenas-Ruano A, Chavez AE, Cossio MJ, Castillo PE, Zukin RS (2012) REST-dependent epigenetic remodeling promotes the developmental switch in synaptic NMDA receptors. Nature neuroscience 15:1382-1390.

Rutkute K, Asmis RH, Nikolova-Karakashian MN (2007) Regulation of neutral sphingomyelinase-2 by GSH: a new insight to the role of oxidative stress in agingassociated inflammation. J Lipid Res 48:2443-2452.

Singh-Manoux A, Kivimaki M, Glymour MM, Elbaz A, Berr C, Ebmeier KP, Ferrie JE, Dugravot A (2012) Timing of onset of cognitive decline: results from Whitehall II prospective cohort study. BMJ 344:d7622.

Singh I, Pahan K, Khan M, Singh AK (1998) Cytokine-mediated induction of ceramide production is redox-sensitive. Implications to proinflammatory cytokine-mediated apoptosis in demyelinating diseases. The Journal of biological chemistry 273:20354-20362. 
Skvarc DR, Dean OM, Byrne LK, Gray L, Lane S, Lewis M, Fernandes BS, Berk M, Marriott A (2017) The effect of N-acetylcysteine (NAC) on human cognition - A systematic review. Neurosci Biobehav Rev 78:44-56.

Tang YP, Shimizu E, Dube GR, Rampon C, Kerchner GA, Zhuo M, Liu G, Tsien JZ (1999) Genetic enhancement of learning and memory in mice. Nature 401:63-69.

Tavallaie M, Voshtani R, Deng X, Qiao Y, Jiang F, Collman JP, Fu L (2020) Moderation of mitochondrial respiration mitigates metabolic syndrome of aging. Proceedings of the National Academy of Sciences of the United States of America 117:9840-9850.

Wang G, Dinkins M, He Q, Zhu G, Poirier C, Campbell A, Mayer-Proschel M, Bieberich E (2012) Astrocytes secrete exosomes enriched with proapoptotic ceramide and prostate apoptosis response 4 (PAR-4): potential mechanism of apoptosis induction in Alzheimer disease (AD). J Biol Chem 287:21384-21395.

White CW, 3rd, Fan X, Maynard JC, Wheatley EG, Bieri G, Couthouis J, Burlingame AL, Villeda SA (2020) Age-related loss of neural stem cell O-GlcNAc promotes a glial fate switch through STAT3 activation. Proceedings of the National Academy of Sciences of the United States of America 117:22214-22224.

Zhang Y, Ji F, Wang G, He D, Yang L, Zhang M (2018) BDNF Activates mTOR to Upregulate NR2B Expression in the Rostral Anterior Cingulate Cortex Required for Inflammatory Pain-Related Aversion in Rats. Neurochemical research 43:681-691.

Zheng J, Xie Y, Ren L, Qi L, Wu L, Pan X, Zhou J, Chen Z, Liu L (2021) GLP-1 improves the supportive ability of astrocytes to neurons by promoting aerobic glycolysis in Alzheimer's disease. Mol Metab:101180. 
bioRxiv preprint doi: https://doi.org/10.1101/2021.06.08.445892; this version posted June 9, 2021. The copyright holder for this preprint (which was not certified by peer review) is the author/funder. All rights reserved. No reuse allowed without permission.

Zhu Y, Carvey PM, Ling Z (2006) Age-related changes in glutathione and glutathione-related enzymes in rat brain. Brain Res 1090:35-44. 


\section{Figure legends}

\section{Figure 1.}

nSMase 2 regulates the ceramide composition in the middle-aged brain

Sphingolipidomics (LC-MS/MS) analysis shows that the levels of all ceramide species are reduced in the nSMase2-deficient cortex (fro/fro, red) compared to heterozygous controls (+/fro, blue). Ceramide concentrations were normalized to lipid phosphate. $\mathrm{N}=3,10$-month old male, ratiopaired $t$-test; $p<0.0001$ for the difference in the levels of $\mathrm{C}_{24: 1}, \mathrm{C}_{18: 0}$, and $\mathrm{C}_{18: 1}$ ceramide. Student's $t$-test and ANOVA.

\section{Figure 2}

\section{Ceramide is mainly increased in astrocytes during aging of the brain}

(A-E) Immunocytochemistry shows that ceramide labeling (red) mainly increases in 10 and 15month old (B-D, arrows) vs. 3-month old heterozygous (+/fro) cortex (A). Ceramide labeling is localized in astrocytes (GFAP, green) and to a lesser extent in microglia (purple in C) and neurons (purple in D). Punctate labeling of ceramide is also visible in close vicinity to astrocytic processes (arrows in B and E) and it is reduced in 15-month old fro/fro mice (E). (F) Quantitation of colocalization using Pearson's coefficient for colabeling of ceramide with markers for astrocytes, (GFAP), microglia (Iba1), and neurons (Neurotrace). N=8. Unpaired $t$-test and ANOVA. 


\section{Figure 3.}

nSMase2-sensitive transcripts are related to oxidative phosphorylation, ribosome biogenesis, synaptic transmission, and memory

(A) Volcano plot shows differentially abundant transcripts in homozygous (fro/fro) vs. heterozygous $(+/$ fro $)$ mouse cortex. Red labels denote increased and green decreased transcripts in fro/fro cortex. The two transcripts decreased most are cytochrome c oxidase subunit VIIa polypeptide 2-like pseudogene (Gm6969) (and ribosomal protein L30 (RPL30) . The transcript most increased is Grin2b (NMDA receptor subunit 2B, arrow). (B) IPA analysis shows that Grin2b is one of a set transcripts encoding proteins important for memory that are increased in fro/fro mice. (C, D) KEGG pathway enrichment analysis of over-represented functional relationships among transcripts increased in fro/fro (CThom) cortex as compared to +/fro (CThet) cortex, showing the top 10 most significant biological pathways. (E) KEGG pathway analysis of oxidative phorphorylation shows the protein assembly in oxidative phosphorylation depicting five mitochondrial complexes containing proteins encoded by transcripts decreased in fro/fro mice. The color legend represents fro/fro vs. +/fro $\log _{2} \mathrm{FC}$ with red denoting upregulation, green denoting downregulation, and grey indicating $\log _{2} \mathrm{FC}$ is zero.

\section{Figure 4.}

Oxidative stress reduces GSH levels and increases nSMase2-mediated ceramide generation in reactive astrocytes

(A) Oxidative stress induced by treatment of heterozygous (+/fro) astrocytes for $1 \mathrm{~h}$ with $200 \mu \mathrm{M}$ tBO2H led to the depletion of GSH (MCB labeling) and increase of ceramide and GFAP labeling, while nSMase2-deficient (fro/fro) astrocytes showed no increase of ceramide or GFAP levels. 
There is no colabeling of GSH with ceramide. (B) Inhibition of nSMase2 with GW4869 (10 $\mu \mathrm{M})$ or restoration of GSH with NAcCys $(500 \mu \mathrm{M})$ prevents ceramide generation induced by oxidative stress $(100 \mu \mathrm{M}$ tBO2H for $2 \mathrm{~h})$.

\section{Figure 5 .}

nSMase2 regulates expression of senescence markers, pro-inflammatory cytokines and Stat3 activation

(A, B) Five-weeks old cultures of primary fro/fro astroctyes show significantly less $\beta$ galactosidase staining than heterozygous (+/fro) astrocytes. $\mathrm{N}=3$. (C-E) Immunoblots for senescence markers p27 and C3b (C), cytokines (IL-1 $\beta$, IL-6, and TNF- $\alpha$ ) (D), and pStat3 and Stat3 (E) in protein prepared from 10-months old male wild type (WT) and nSMase2-deficient (fro/fro) cortex. $\mathrm{N}=3$. Unpaired $t$-test.

\section{Figure 6.}

\section{nSMase2 regulates Grin2b transcription networks}

(A) IPA analysis of the set of differentially abundant transcripts shows the most enriched mechanistic network, including motor behavioral coordination-associated networks and processing of RNA. The legends of network and path designer shapes have been listed underneath of networks, red color denotes activated function and blue color denotes inhibited function. (B) Grin2b, Grin2a, and BDNF were predicted to be coordinatedly upregulated in fro/fro cortex. 


\section{Figure 7.}

\section{nSMase2 deficiency increases Grin2b expression}

(A) Synaptic network for receptor cell signaling upregulated in fro/fro cortex, including NMDA receptor (circled in red) containing Grin2a and Grin2b subunits. (B) Protein analysis of Grin2b in fro/fro cortex as compared to wild type controls. $\mathrm{N}=3$ pairs (littermates from 3 dams), two tails paired $t$-test.

\section{Figure 8.}

nSMase2 deficiency reduces the number of exosomes that decrease Grin2b

(A) The upstream regulator analysis by IPA showing transcripts downstream of REST and miR223-3p that decreased in fro/fro cortex. The inhibited function of REST and miR-223-3p as shown in blue color positively activate downstream Grin2b. The transcripts downstream of REST and miR-223-3p are positively associated with neuron number, long-term potentiation, epilepsy, behavioral conditioning, and memory. (B) qPCR for miR-223-3p in exosomes from fro/fro and +/fro brain. Plot shows fold-change when compared to exosomal house keeping miRNA (miR30c-5p). $\mathrm{N}=3$, unpaired $t$-test. (C, D) Nanoparticle tracking analysis (Zetaview) of extracellular vesicles from +/fro and fro/fro cortex. The mumber of exosomes are shown as per militer (ml) of PBS or per gram (gm) of brain tissues $\mathrm{N}=3$. Unpaired $t$-test. 


\section{Figure 9.}

Hypothetical model for regulation of neuronal function by nSMase2 in astrocytes and agingassociated astrosomes (AAAs)

Oxidative stress leads to the generation of reactive oxygen species (ROS) in astrocytes and in neurons. In astrocytes, GSH is secreted to provide GSH precursors to neurons. In neurons, GSH resynthesized from astrocytes-derived precursors neutralizes ROS to maintain neuronal function. Increased oxidative stress during aging leads to reduction of GSH levels in astrocytes and activation of nSMase2, which induces formation and secretion of aging-associated astrosomes (AAAs) that suppress neuronal activity. In nSMase2-deficient (fro/fro) mice, lack of ceramide elevation prevents formation of AAAs, and in turn, preserves neuronal function.

Table 3

Positively behavior related diseases or Function Analysis

\begin{tabular}{lllll}
\hline Diseases or functions & $\boldsymbol{p}$-value & Predicted & Activation & \#Molecules \\
Annotation & & activation & z-score & \\
& & state & & \\
\hline Cognition & $4.04 \mathrm{E}-23$ & Increased & 2.995 & 118 \\
Learning & $1.76 \mathrm{E}-20$ & Increased & 3.008 & 106 \\
Conditioning & $2.47 \mathrm{E}-09$ & Increased & 2.976 & 46 \\
Memory & $1.26 \mathrm{E}-12$ & Increased & 3.591 & 64 \\
\hline
\end{tabular}




\section{Figure 1}



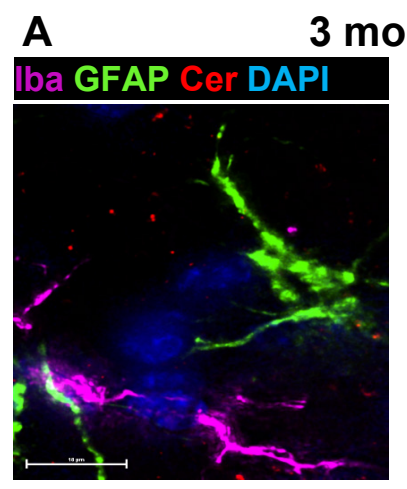

C

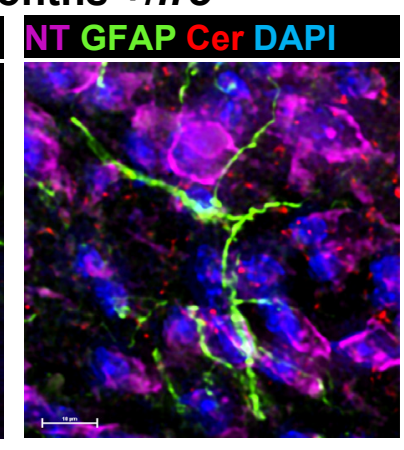

B 10 months +/fro


15 months +/fro



D

15 months +/fro
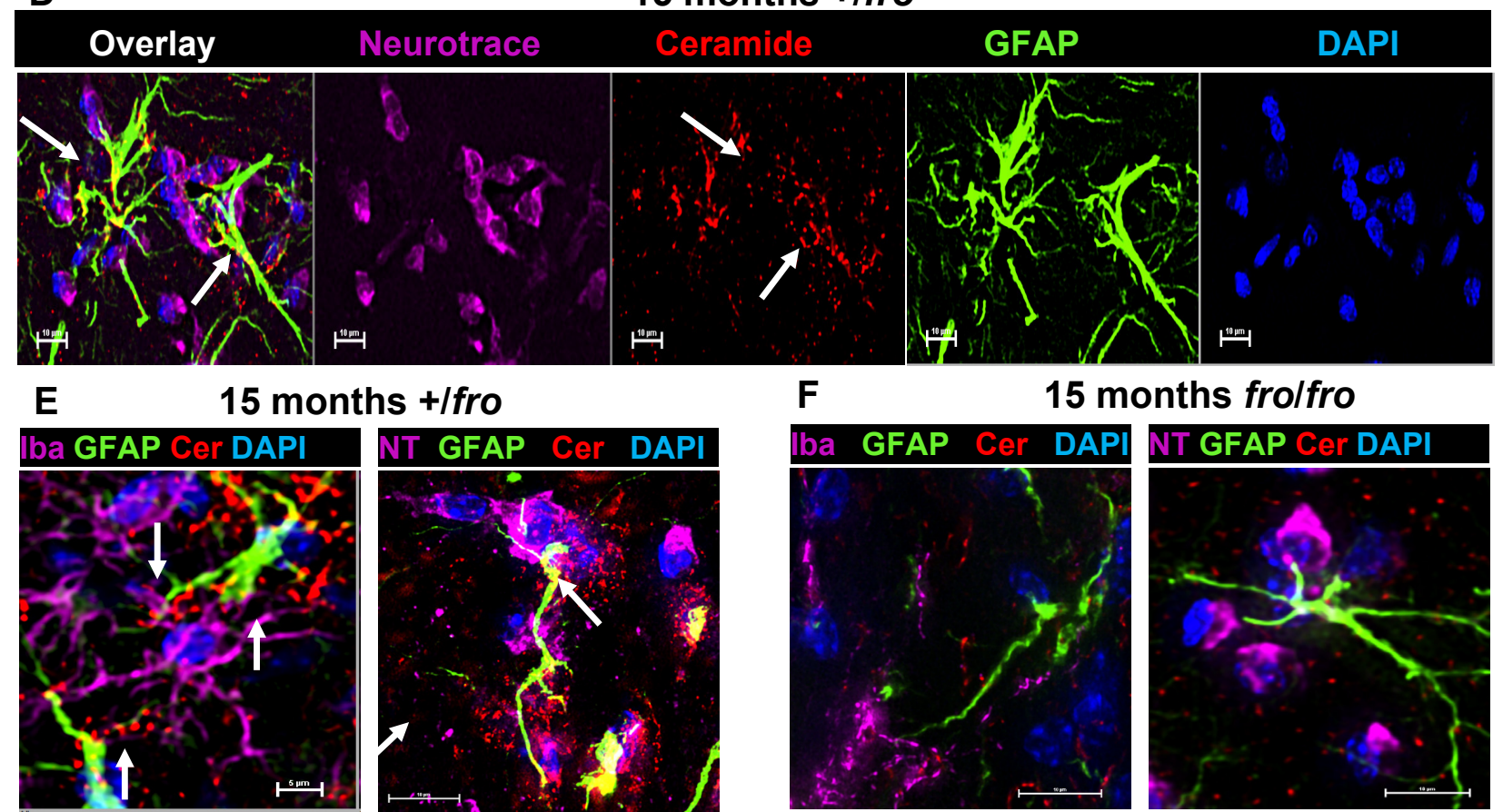

G






\section{Figure 3}

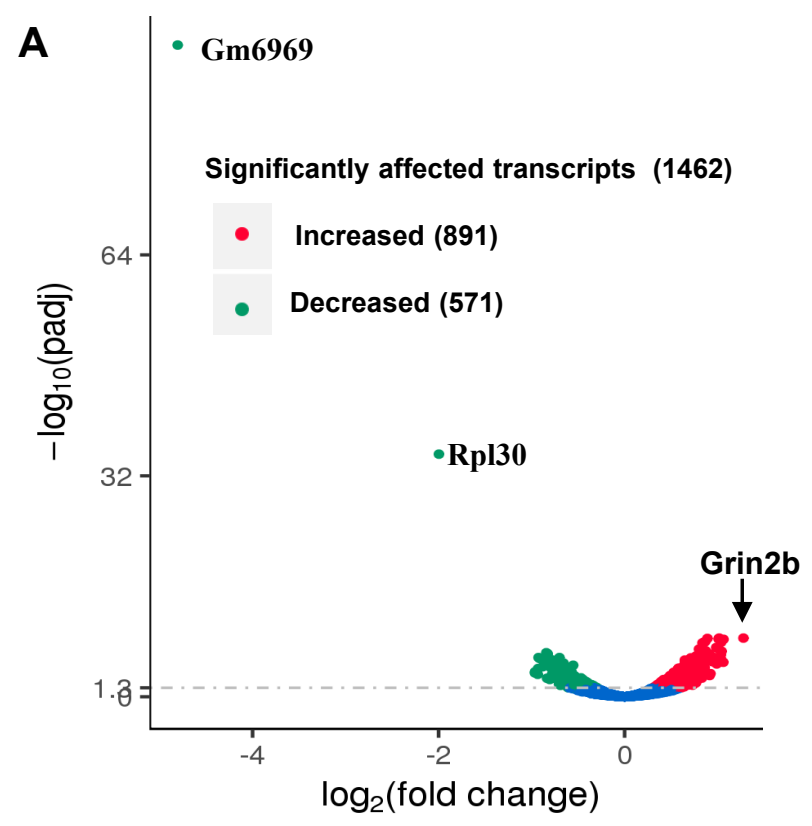

B

\begin{tabular}{|c|c|c|c|}
\hline \multicolumn{4}{|c|}{ Memory predicted to be increased (z-score 3.6) } \\
\hline \multicolumn{4}{|c|}{\begin{tabular}{l|l|l}
$\begin{array}{l}\text { Genes in } \\
\text { dataset }\end{array}$ & Prediction & Expr Log Ratio
\end{tabular}} \\
\hline \multicolumn{4}{|c|}{\begin{tabular}{l|l|l|} 
GRINDR & Increased & 1278
\end{tabular}} \\
\hline \multicolumn{4}{|c|}{ Increased } \\
\hline \multicolumn{4}{|l|}{ NF1 } \\
\hline \multicolumn{4}{|l|}{ NOTCH1 } \\
\hline \multicolumn{4}{|l|}{ IGF2R } \\
\hline \multicolumn{4}{|l|}{ ERBB4 } \\
\hline \multicolumn{4}{|l|}{ CREBBP } \\
\hline \multirow{2}{*}{$\begin{array}{l}\text { GSK3B } \\
\text { LRRC7 }\end{array}$} & Increased & 0.551 & $\uparrow$ \\
\hline & Increased & 0.536 & $\uparrow$ \\
\hline ADCY8 & Increased & 0.532 & $\uparrow$ \\
\hline MTOR & Increased & 0.516 & $\uparrow$ \\
\hline
\end{tabular}

C

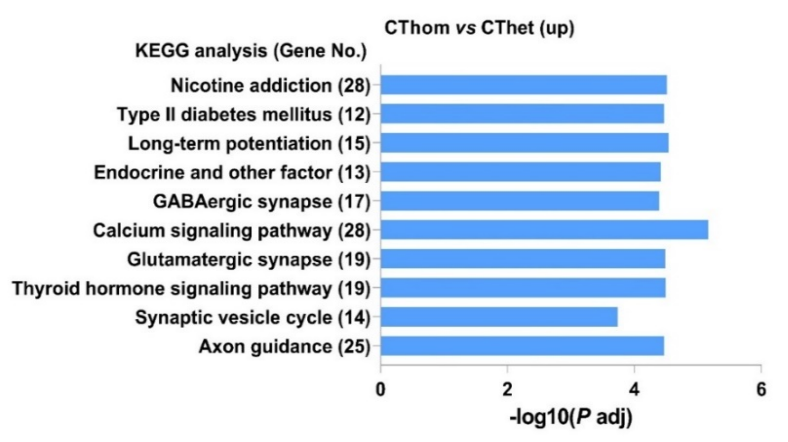

D
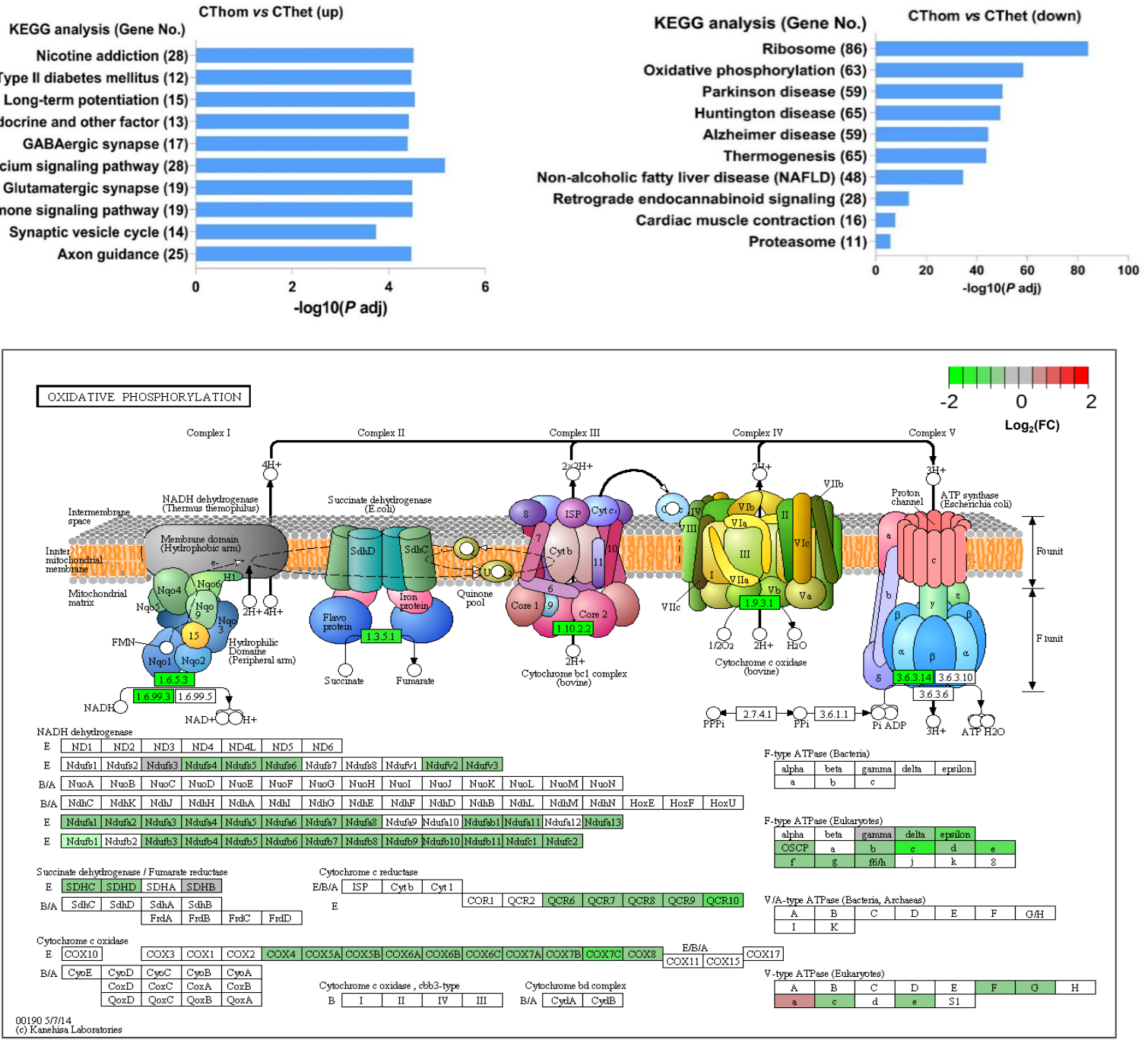
Figure 4

A

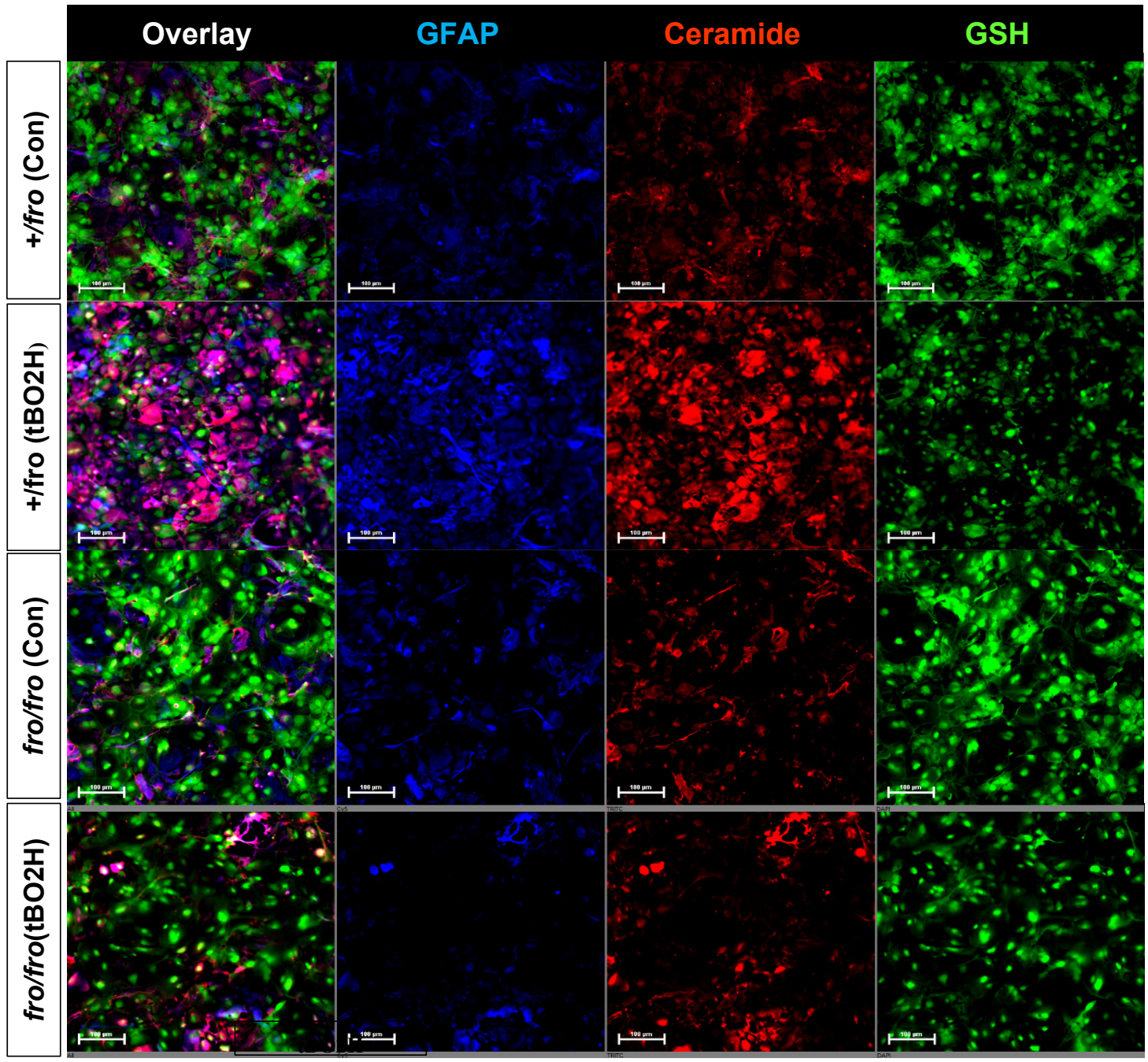

B

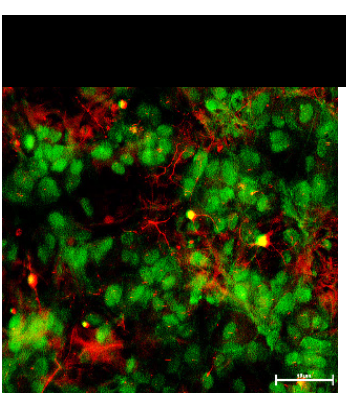

Con
Ceramide



tBO2H
GSH



tBO2H+GW4869

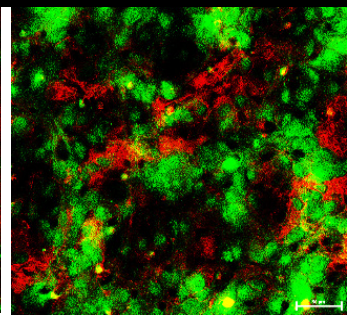

tBO2H+NAc 
Figure 5

A
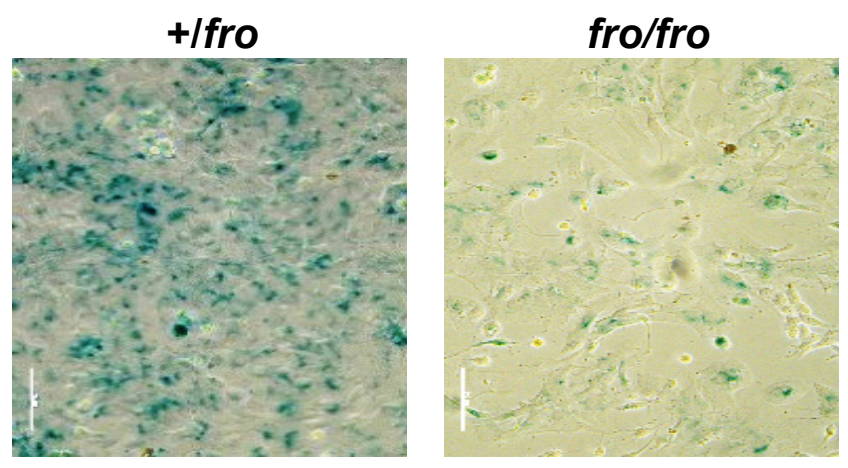

B

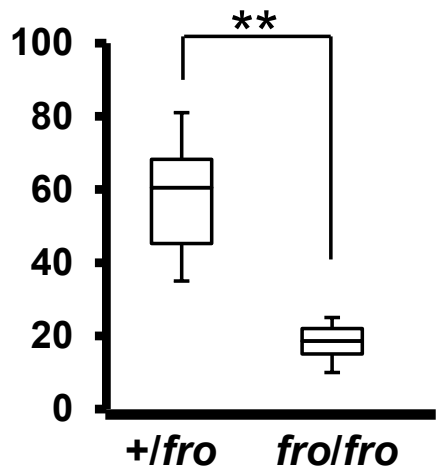

C
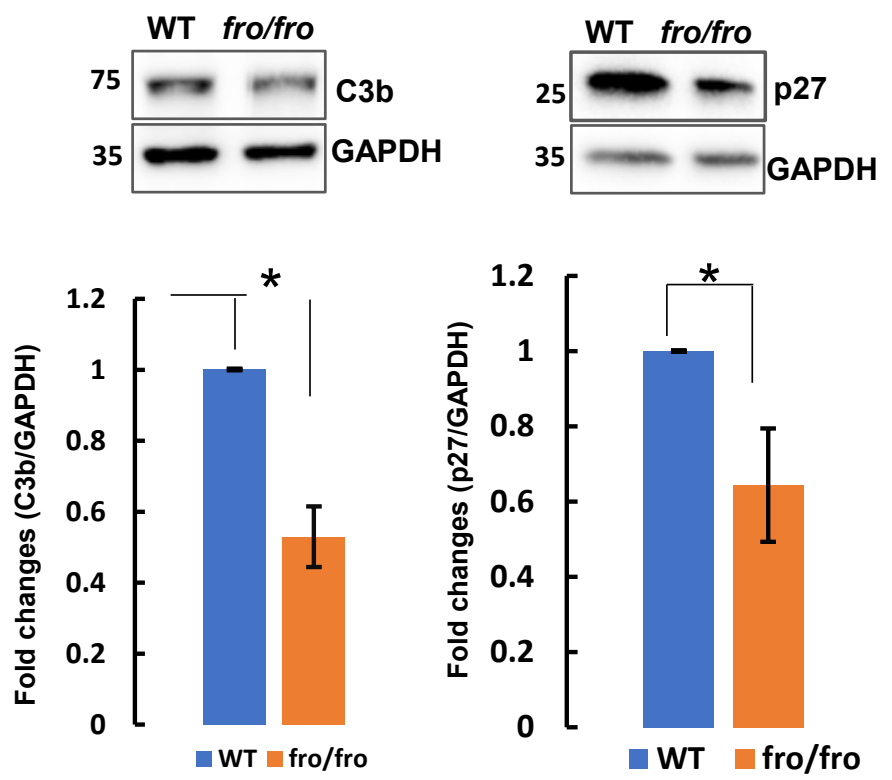

D
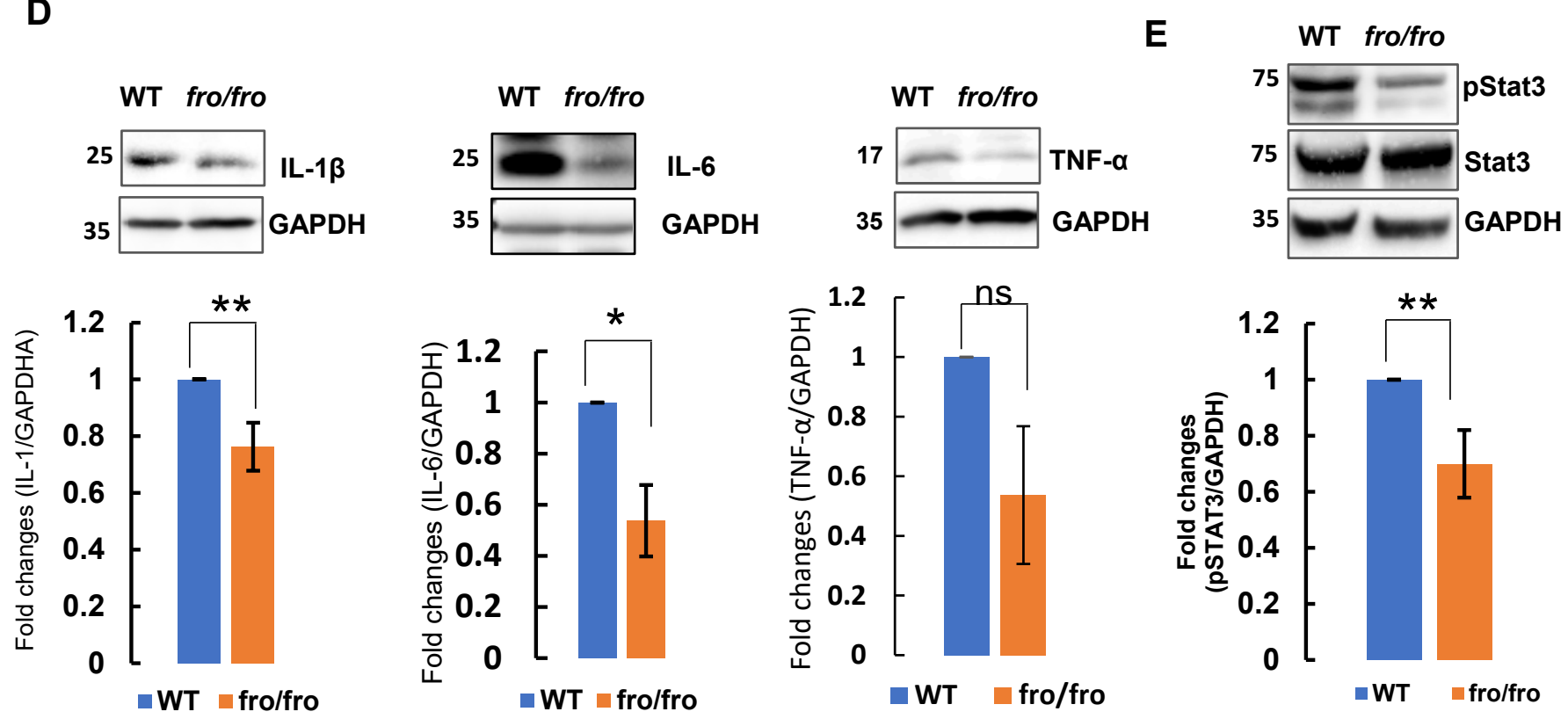


\section{Figure 6}

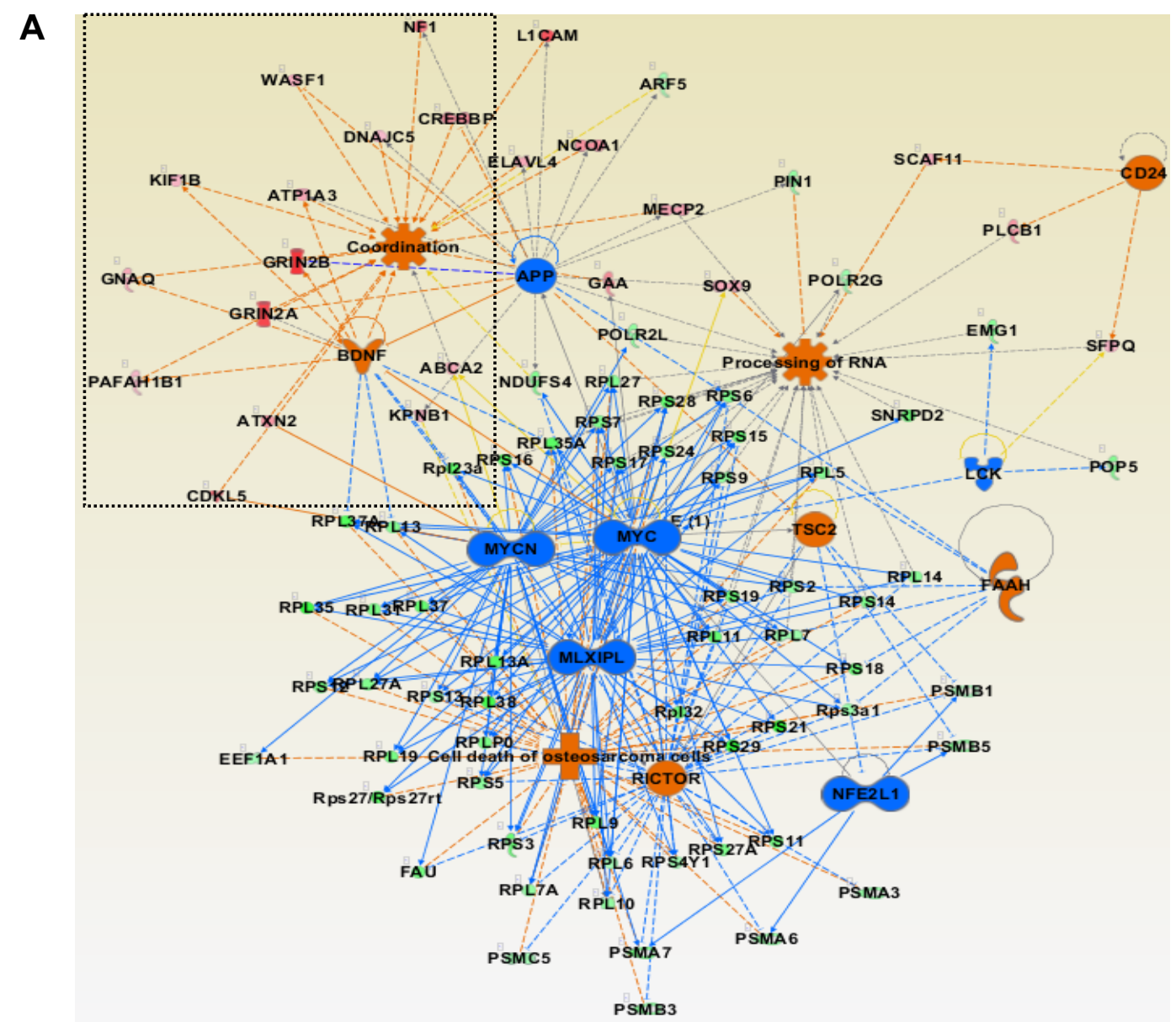

B
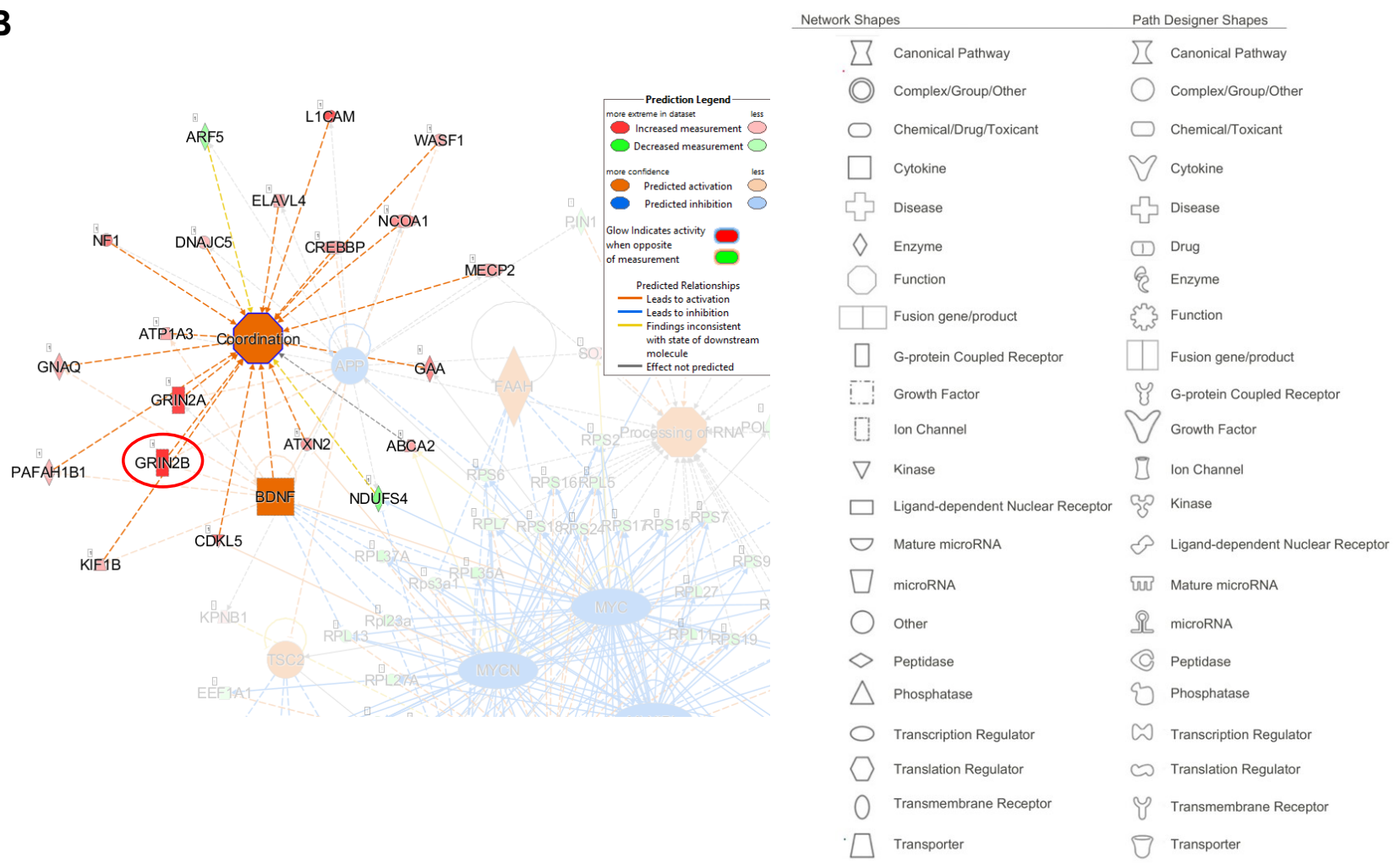


\section{Figure 7}

A

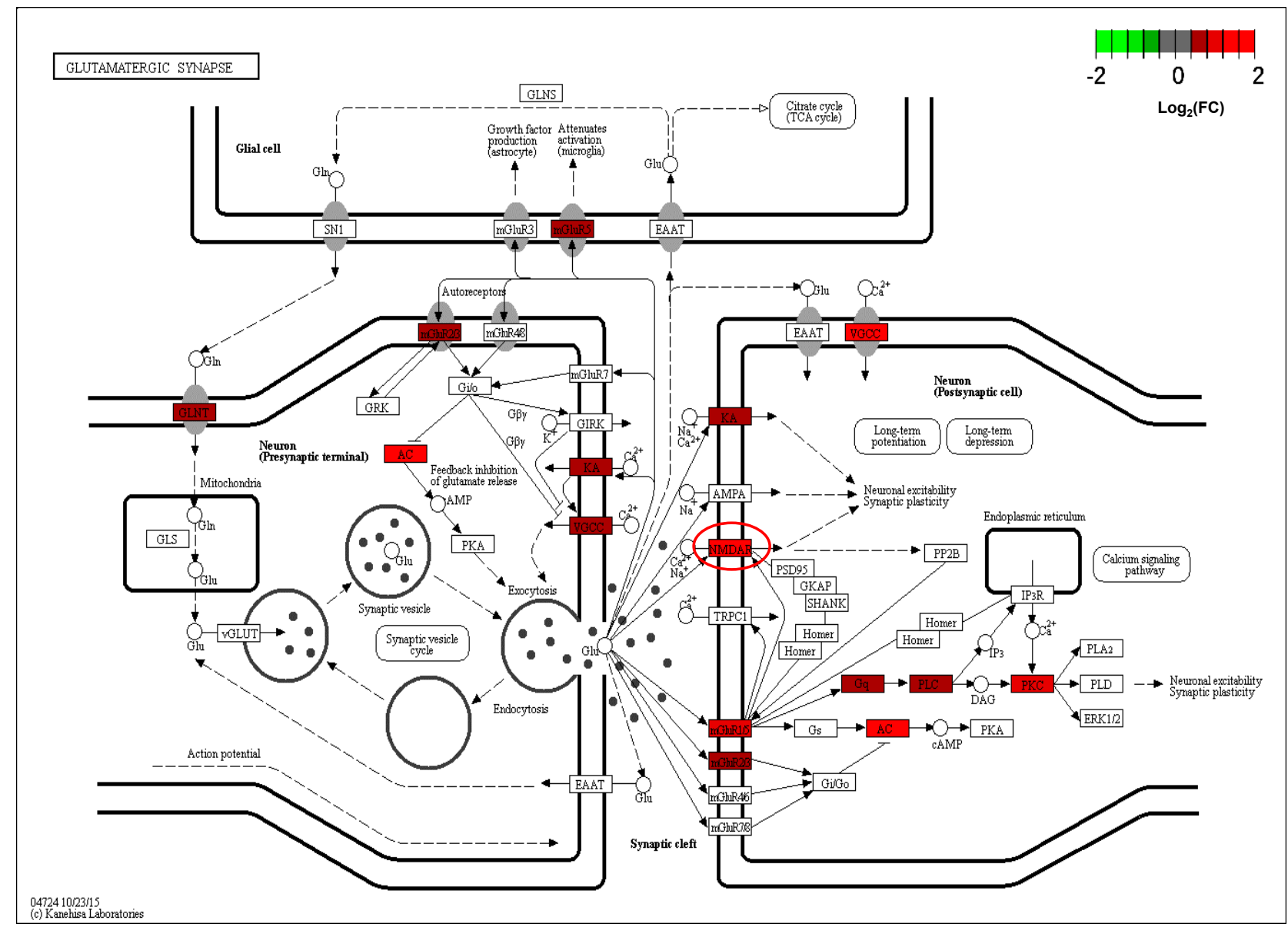

B
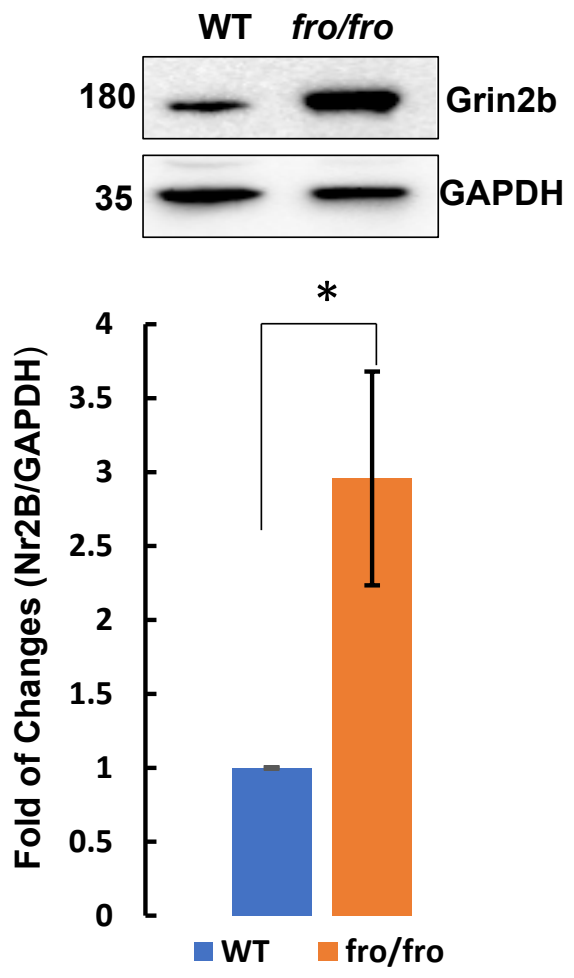


\section{Figure 8}

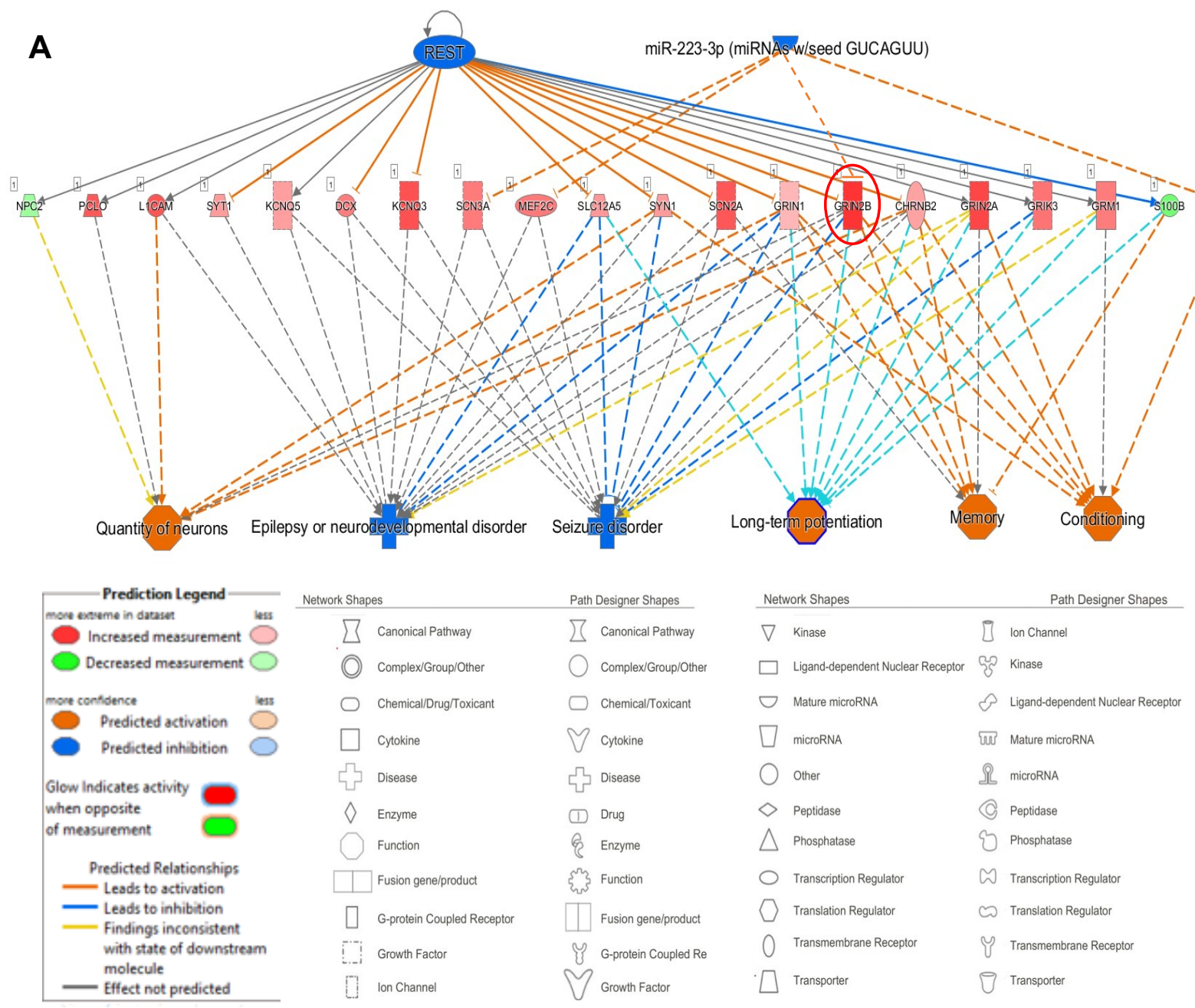

B

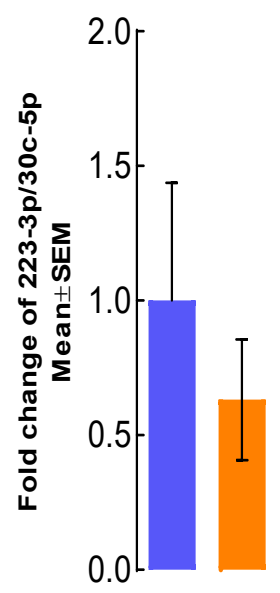

C

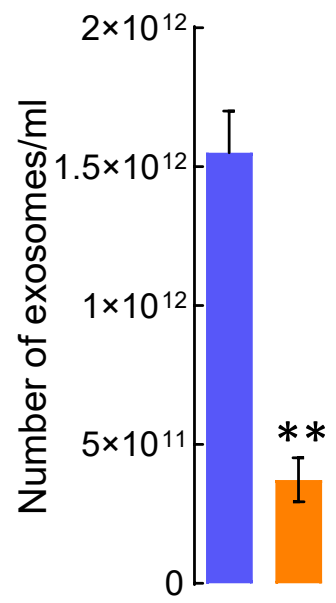

D

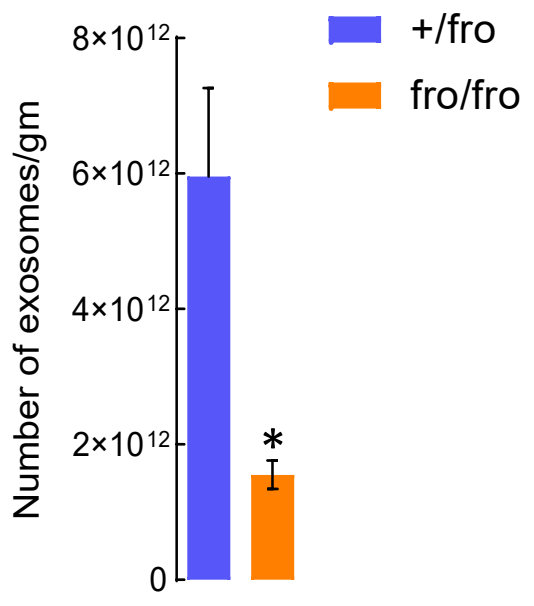




\section{Figure 9}

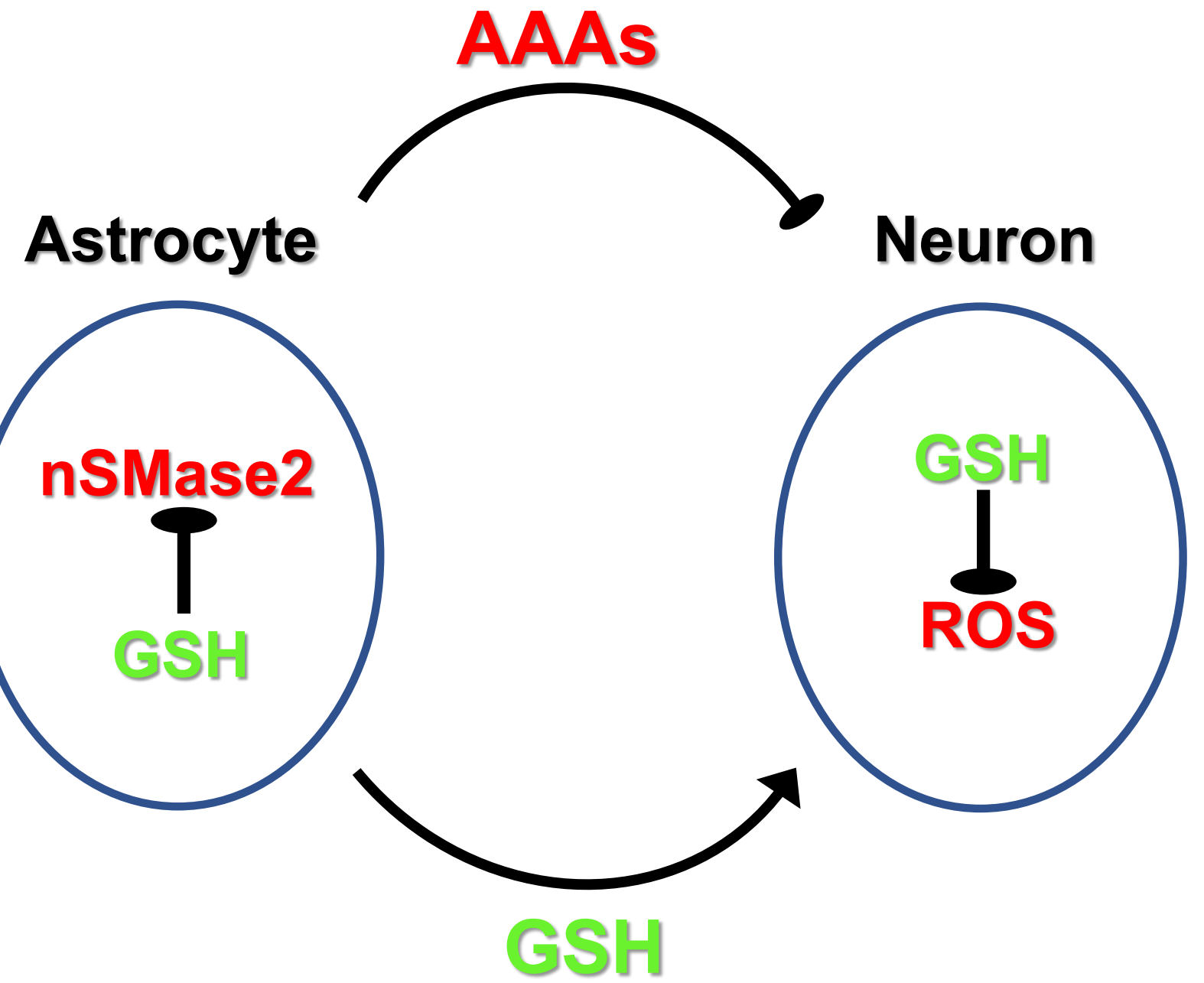

